\title{
CONCEPT OF A FORMAL SYSTEM AIMED AT MODELLING BIOLOGICAL PROCESSES IN CELL
}

\author{
JAROSŁAW KACZMAREK \\ Institute of Fluid-Flow Machinery, Polish Academy of Sciences, \\ J. Fiszera 14, 80-952 Gdańsk, Poland \\ (Received 19 February 2001)
}

\begin{abstract}
In this paper a concept of a formal system aimed at modelling biological processes in cell is introduced. The aim of this formulation is to provide a theoretical basis for numerical simulations of the biological processes taking into account accessibility to various levels of details and possible full complexity of phenomena associated with them. The main segment of this formal system is a method of multiscale modelling called here the collection of dynamical systems with dimensional reduction. This approach allows one to integrate various mathematical methods applied in cell biology. It is especially related to continuum mechanics and molecular dynamics. Such a formal mathematical approach is supplemented by a set of notions which characterizes specific properties of biological systems. They are: the molecular recognition, integrity property for a chain of chemical reactions and molecular reactive structures. Nanoscale models of molecules are suggested to be a central segment of the multiscale modelling and are designed to direct cooperation with molecular dynamics. They are aimed at describing multimolecular processes in cell. It is shown that the nanoscale level of description is convenient for modelling molecular motors. Status of the present stage of formulation of the formal system is discussed taking into account further development directions.
\end{abstract}

\section{INTRODUCTION}

Complexity of biological systems leads to application of various mathematical methods in modelling of them. In particular we can distinguish two important theoretical directions considered in modelling of biological processes. The first one is based on continuum models [1], Then, more averaged properties of biological systems are usually described. The second one is connected with discrete methods related to atomic level of modelling represented by equations of molecular dynamics [2], Methods of molecular dynamics are supported by quantum mechanics. However, quantum mechanics is also applied independently in modelling in biology. Discussed theoretical directions lead to miscellaneous methods of numerical simulations applied in biology. Such approaches embody the fact that biological structures are connected with processes related to various scales.

Considerations of this paper are concentrated around concepts of integration of the molecular dynamics and continuum mechanics. Consequently, we do not discuss problems related to quantum mechanical modelling of biological processes.

We could pose a general question: what we would like to attain by numerical simulations in biology, neglecting at this moment current possibility of realization of them. An interesting aim is modelling and numerical simulations of the whole cell as a system of processes. Obstacles lying on way leading to realization of such an aim are serious. Molecular dynamics is able to simulate behaviour of single proteins [3], Biological processes in cell are usually multimolecular. Thus, 
large number of atoms and complicated interactions, especially with solvent, makes difficult to use molecular dynamics directly to modelling processes. On the other hand continuum models describe processes in a very averaged way neglecting their real complexity [1], Finally, not all processes are well understood taking into account current state of molecular biology. However, despite of these difficulties, the question what kind of mathematical approach would be appropriate for modelling cell processes is discussable.

In order to use mathematical methods for modelling biological systems in a systematic way we would like to have at our disposal a formal system. Let us note that a formal system was introduced for mechanics. Such a system is called the rational mechanics and has been developed for many years [4], As a result we have obtained considerable progress in systematization of mathematical methods applied especially in mechanics of continuum [4], This creates convenient theoretical basis for application of various numerical simulations in mechanics.

Considerations related to a formal system aimed at modelling biological processes in cell need discussion of characteristic properties of them. It would be convenient to define precisely biological molecular structures. However, the most fundamental laws which govern their evolution are perhaps not entirely known. Therefore, we confine ourselves only to characterization of such structures here, taking into account some aspects important for discussed in this paper stage of modelling.

Biological structures have considerably differentiated properties manifested at various scales. They represent possibilities of bearing even strong loadings and, on the other hand, they represent very fine processes such as precise chemical reactions in water. The fine processes can be realized owing to stabilization of conditions by a part of this structure which is able to bear stresses. Such a structure is usually connected with a larger scale than the chemical reactions.

Elementary mechanisms governing evolution of the biological structures are submolecular. However, characteristic processes are usually multimolecular which especially accentuate multiscale nature of them.

Finally, all processes of biological systems are interacting. They are integrated within larger units which are open systems interchanging mass and energy with an environment.

Mathematical methods in biology are developed for many years. In particular, biological cybernetics and works of Haken [5] accentuate important role of dynamical systems in mathematical modelling in biology. However, current state of these approaches is not connected with elaboration of formal system similar to that one considered in mechanics. Such a situation seems to be caused by lack of formalization of scale of averaging assumed in modelling of biological processes.

Consequently, in the case of modelling biological systems we should be able to describe processes at various scales. This directly follows that formalization of scale is an important aspect of the discussed formal system. Furthermore, we see that integration of continuum mechanics as well as models representing atomic or even subatomic level should be carried out within the formal system. Such an uniform approach would be able to express more averaged properties of biological systems basing on smaller scale interpretation. 
The long range aim of the constructed formal system is to create a theoretical basis for numerical simulations of cell processes viewed as an integrated system. Then, the question relating to range of validity of particular equations applied is of great importance. Indeed, in complex system of phenomena we have to do with changing external conditions influencing evolution of a subsystem considered. Then, in order to apply correctly equations describing its evolution we have to know range of validity of these equations. Determination of the range of validity of equations applied can be given by some critical conditions. Such a situation enables us to change the description during simulations in the case when the critical conditions are violated. Thereby, knowledge of range of validity of applied equations is a necessary condition for reliable numerical simulations of larger systems. Therefore, this aspect should be also expressed in formulation of our formal system.

The aim of this paper is to introduce a concept of a formal system which takes into account multiscale properties of processes described, possibility of integration of various mathematical methods related to modelling biological molecular structures as well as specificity of biological processes in cell.

In this paper we introduce the collection of dynamical systems with dimensional reduction as a theory which realizes requirements related to formalization of scale within mathematical methods of modelling. This is a multiscale description which contains continuum mechanics as a particular case and problem of scale of averaging is viewed there as the most important. This is a good candidate to be the main part of our formal system. However, such a mathematical framework needs additional system of notions which would be able to distinguish biological structures from many others, taking into account their specificity.

Notions defined for characterization of biological structures are introduced here by definitions of the molecular recognition, integrity property for a chain of chemical reactions and molecular reactive structures.

The molecular recognition enables us to consider necessary conditions for realization of precise synthesis which happens in biological systems. The integrity property for a chain of chemical reactions allows one to discuss integrity within a more complex system of chemical reactions. Molecular reactive structures are designed to model more stable with respect to chemical composition molecular structures which can cooperate with chemical reactions.

In this paper particular role of the nanoscale description of single molecules is accentuated. We have discussed above a problem with modelling biological processes which have elementary mechanisms at submolecular level, however themselves are multimolecular. Discussed in this paper nanoscale models are just designed to modelling multimolecular processes owing to considerable reduction of degrees of freedom in comparison with molecular dynamics dynamical systems.

Nanoscale models could also be viewed as a starting point for derivation of more averaged models by means of a dimensional reduction procedure. 


\section{COLLECTION OF DYNAMICAL SYSTEMS WITH DIMENSIONAL REDUCTION}

\subsection{Dimensional reduction procedure}

General aim of formulation of the collection of dynamical systems with dimensional reduction is to elaborate methods of dimensional reduction of an elementary dynamical system (EDS). The elementary dynamical system represents description of physical processes on an assumed elementary level and is characterized usually by considerable complexity. By means of the dimensional reduction we obtain a simplified model based on theoretical foundations provided by the $E D S$.

Let $\varphi$ be variables of EDS and $\mathbf{f}$ represents external interactions acting on such a system. Then, we introduce the elementary dynamical system in the form

Transition to a larger scale of averaging is connected with a simplification of this model, corresponding to reduction of degrees of freedom. Our concept of this simplification consists in division of the elementary dynamical system (1) into subsystems. Subsystems are distinguished by determination of groups of variables $\varphi_{h}=\left\{\varphi_{h \alpha}\right\}, \alpha \in I_{A h}$. Then, our initial variable takes the form $\varphi=\left\{\varphi_{h}\right\}, h \in I_{P}=\{1, \ldots, P\}$, where $P$ is a number of subsystems. Selection of variables into groups is introduced by means of set of indexes $I_{A h}$ which number components of $\varphi$ which belong to the $h$-th group. In general, we admit the case when the set $I_{A h}$ can be varying during evolution of a given subsystem.

Determination of subsystems allows one to introduce new variables. They represent a reduced number of degrees of freedom and describe approximately behaviour of each subsystem. New variables are introduced by a mapping $\pi_{T}: V_{T} \rightarrow \bar{V}_{T}$ which transform processes $\varphi(t) \in V_{T}$ determined on a time interval $T$ into processes determined on the reduced level of description $\mathbf{d}(t) \in \bar{V}_{T}$.

External interactions acting on the dimensionally reduced dynamical system have to correspond to those ones introduced for the elementary dynamical system. Such a correspondence is introduced by a mapping $\pi_{f T}: \mathcal{F}_{T} \rightarrow \overline{\mathcal{F}}_{T}$ which transforms processes of external interactions.

The dimensional reduction procedure $D R=\left\{\boldsymbol{\Pi}_{T}, \pi_{\mathrm{f}}, S D S\right.$, app $\}$ consists of four elements. Determination of new variables and external interactions with the help of $\pi_{T}$ and $\pi_{f T}$ is a first step in postulating a skeletal dynamical system $\operatorname{SDS}$. The $S D S(\mathbf{C})$ represents a set of dynamical systems with elements depending on constants C. Final form of the dimensionally reduced dynamical system $R D S$ is obtained by identification of the best constants $\overline{\mathbf{C}}$ by comparison of solutions of the elementary dynamical systems and solutions depending on $\mathbf{C}$ which are obtained from equations of the skeletal dynamical systems. Then we obtain $\operatorname{RDS}=\operatorname{SDS}(\overline{\mathbf{C}})$. The set of all methods of approximation and identification applied for obtaining $\overline{\mathbf{C}}$ is denoted by app and is viewed as fourth component of the dimensional reduction procedure. 


\subsection{Balance of mass and energy for collection of dynamical systems}

In order to postulate a form of the skeletal dynamical system we have to introduce a set of assumptions which enable to transfer fundamental physical laws expressed by balance of mass and energy equations into the reduced level. They are transferred from the EDS level.

Let $\mathcal{M}_{\Pi}=\left\{\left\{\varphi_{h}\right\}\right\}, h \in I_{P}$ stand for space of solutions of the elementary dynamical system with distinguished groups of variables $\varphi_{h}$ related to $h$-th subsystem. We introduce the following set of assumptions:

1. There exists a function $\bar{m}_{h}\left(\varphi_{h}\right)=\left\{m_{h 1}, \ldots, m_{h \beta h}\right\}$ which assigns a set of masses for the $h$-th subsystem. The total mass of this subsystem is then $m_{h}=\sum_{i} m_{h i}$. We have also that $\sum_{h} \beta_{h}=N$, where $N$ is the total number of masses in the whole system. The function $\widetilde{m}: \mathcal{M}_{\Pi \rightarrow} \rightarrow R^{P}$ with property $\widetilde{m}\left(\left\{\varphi_{h}\right\}\right)=\left\{m_{h}\right\}$ determines distribution of masses in subsystems and $m: \mathscr{M}_{\Pi \rightarrow} \rightarrow$, $m\left(\left\{\varphi_{h}\right\}\right)=\sum m_{h}$ determines the total mass related to (1).

2. There exists a function $\widetilde{E}: \mathscr{M}_{\Pi} \rightarrow R^{P}, \widetilde{E}\left(\left\{\varphi_{h}\right\}\right)=\left\{E_{h}\right\}$ which determines distribution of energy assigned to subsystems and $E: \mathcal{M}_{\Pi} \rightarrow R, E\left(\left\{\varphi_{h}\right)\right\}=\sum_{h} E_{h}$ determines the total energy related to (1).

3. There exists a family of mappings $J_{i j}: \mathscr{M}_{I} \rightarrow R, i, j \in I_{P}, J_{i j}\left(\left\{\varphi_{h}\right\}\right)=J_{i j}$ called flux of mass from $j$-th subsystem to $i$-th subsystem and $J_{i j}+J_{j i}=0, J_{i i}=0$.

4. There exists a family of mappings $W_{i j}: \mathcal{M}_{\Pi} \rightarrow R, i, j \in I_{P}, W_{i j}\left(\left\{\varphi_{h}\right\}\right)=W_{i j}$ called flux of energy from $j$-th subsystem to $i$-th subsystem and $W_{i j}+W_{j i}=0, W_{i i}=0$.

5. A source of mass is determined by a function $c: \mathcal{M}_{\Pi \rightarrow} \rightarrow R^{P}, c\left(\left\{\varphi_{h}\right\}\right)=\left\{c_{i}\right\} . c_{i}=\pi_{i}^{\circ} c\left(\left\{\varphi_{h}\right\}\right)$ can be considered for each subsystem of the whole system and stands for a source of mass in the $i$-th subsystem.

6. A source of energy is determined by a function $R: \mathcal{M}_{\Pi} \rightarrow R^{P}, R\left(\left\{\varphi_{h}\right\}\right)=\left\{R_{i}\right\}$. $R_{i}=\pi_{i} \circ R\left(\left\{\varphi_{h}\right\}\right)$ can be considered for each subsystem of the whole system and stands for a source of energy in the $i$-th subsystem.

Let $I_{G} \in I_{P}$ be a set of indexes which distinguishes a group of subsystem. Then, $I_{Q}=I_{P}-I_{G}$ determines subsystems which are external with respect to our group.

By means of above introduced assumptions we are able to carry out analysis of interchange of mass between subsystems as well as to consider possible sources of mass which appear within subsystems. This leads to expressing the balance of mass equation for collection of dynamical systems in the following form

$$
\sum_{i \in I_{G}}\left(\dot{m}_{i}-c_{i}\right)+\sum_{i, j \in I_{G}} J_{i j}+\sum_{i \in I_{G}, j \in I_{O}}\left(J_{i j}+J_{j i}\right)+\sum_{i, j \in I_{O}} J_{i j}+\sum_{i \in I_{O}}\left(\dot{m}_{i}-c_{i}\right)=0 .
$$

Total sum of masses interchanged between subsystems, within the distinguished group by $I_{G}$, without any interchange with an external subsystems is equal to zero. Consequently, we obtain then $\sum_{i, j \in I_{G}} J_{i j}=0$.

Option of $I_{G}$ is arbitrary. Accordingly, we can express the balance of mass equation connected with an arbitrary group of subsystems represented by $I_{G}$ with the help of the formula 


$$
\sum_{i \in I_{G}}\left(\dot{m}_{i}-c_{i}+\sum_{j \in I_{O}} J_{i j}\right)=0
$$

The terms $J_{i j}$ describe interchange of mass with an external system indexed by elements of $I_{O}$. As a result the equation (3) is not entirely determined. This, in turn, necessitates introducing an additional condition

$$
J_{i j}=\bar{J}_{i j}, \quad j \in I_{O},
$$

where $\bar{J}_{i j}$ is given and expresses an assumed form of efflux of mass.

The balance of energy equation has similar structure as the balance of mass equation and is given by

$$
\sum_{i \in I_{G}}\left(\dot{E}_{i}-R_{i}+\sum_{j \in I_{O}} W_{i j}\right)=0
$$

with additional conditions

$$
W_{i j}=\vec{W}_{i j}, \quad j \in I_{O},
$$

where $\bar{W}_{i j}$ represents an assumed form of efflux of energy.

Let us note that $E_{i}$ and $W_{i j}$ depend, in general, on state of the whole system in accordance with assumptions 2 and 4 .

Balance of mass and energy equations given by (3)-(6) are a starting point for postulating the skeletal dynamical system. This is realized by option of new variables and representations, of functions which appear in (3)-(6). These functions are parameterized then by a set of constants which should be next identified.

\subsection{Continuum skeletal dynamical system}

Wide application of continuum mechanics in biology necessitates discussion of way of appearing of this theory within the collection of dynamical systems. Foundations of continuum mechanics has been discussed by many authors, see for instance [6, 4],

We would like to introduce fundamental notions of continuum mechanics in connection with the elementary dynamical system. Continuum is defined by means of geometrical terms. Therefore we introduce an additional assumption related to properties of subsystems distinguished within $E D S$ as follows:

Geometrical objects of various dimensions can be assigned to each subsystem by means of mappings $G_{x}: \mathcal{M}_{\Pi} \rightarrow E_{e}{ }^{P}, G_{L}: \mathcal{M}_{\Pi} \rightarrow\left(2^{E_{e}}\right)^{P}, G_{S}: \mathcal{M}_{\Pi} \rightarrow\left(2^{E_{e}}\right)^{P}, G_{V}: \mathcal{M}_{\Pi} \rightarrow\left(2^{E_{e}}\right)^{P}$, where $2^{E e}$ stands for family of all subsets of the Euclidean space $E_{e}$. The map $G_{x}$ assigns some distinguished points to subsystems, $G_{L}$ introduces one-dimensional, $G_{s}$ two-dimensional, $G_{V}$ three-dimensional geometrical objects considered as subsets of $E_{e}$ and accompanied by distinguished subsystems.

Introduced assumption enables discussion of geometrical objects associated with EDS. In particular, we can discuss position vectors related to $E D S$ by means of mappings $G_{x}$. This gives 
a possibility of considering kinematics and other elementary notions of continuum mechanics in relation to the elementary dynamical system.

For defining a body we consider the mapping $G_{v}\left(\left\{\varphi_{h}\right\}\right)=\left\{K_{h}\right\}$, where $K_{h}$ is a three-dimensional subset of $E_{e}$. Let $\mathcal{K}=\left\{K_{h}, h \in I_{P}\right\}$ and $\mathscr{M}_{K}=\{\mathscr{K}\}$ stand for all families of $K_{h}$ obtained by means of $G_{v}$. Then, $G_{v}: \mathcal{M}_{I I} \rightarrow \mathcal{M}_{K}$. We assume also that int $K_{g} \cap$ int $K_{h}=0, g, h \in I_{P}$, where int is operation of taking interior of a set.

\section{Definition 1:}

The body associated with the elementary dynamical system $\dot{\varphi}=L(\varphi, \mathbf{f})$ is defined with the help of mapping $G_{V}$ as $\mathcal{B}_{\varphi}=\bigcup_{h \in I_{p}} K_{h}$.

Deformation is connected with evolution of points with respect to reference configuration. In order to describe deformation we consider the function $G_{x}$ which assigns a distinguished point $\chi_{\mathrm{h}}$ to each subsystem. Consequently, we have that $G_{x}: \mathscr{M}_{\Pi} \rightarrow\left\{\left\{\chi_{h}\right\}\right\}$ We interpret $\left\{\chi_{\mathrm{h}}\right\}$ as distinguished positions associated with subsystems.

Let $H_{\chi h}=\left\{\chi_{m}, m \in I_{h}{ }^{a}\right\}$ and $V_{a h}$ be a linear space. The set $I_{h}^{a}$ represents indexes of subsystems $K_{m}$ which interact with $K_{h}$. Then, we introduce the function $a_{h}:\left\{H_{\chi h}\right\} \rightarrow V_{a h}$ and $a:\left\{\left\{H_{\chi h}\right\}, h \in I_{P}\right\} \rightarrow\left\{\left\{a_{h}\left(\left\{\chi_{m}\right\}\right)\right\}, h \in I_{P}\right\}$ as a function of kinematical dependence between subsystems. By this function the gradient of deformation and strain tensor can be introduced.

The space $\vec{V}_{D}=\left\{\left\{\chi_{h}, a_{h}\right\}, h \in I_{P}\right\}$ characterizes deformation determined by a finite number of parameters. Let us define the space $V_{K}$ of deformation functions $\chi_{K}$ of the body $\mathcal{B}$ with respect to a given configuration $\chi$ as $V_{\kappa}=\left\{\chi_{\kappa}: \chi_{\kappa}=\lambda \circ \kappa^{-1}, \lambda, \kappa \in \mathcal{C}\right\}$ in accordance with classical formulation of continuum mechanics [41. Let furthermore, $\alpha_{\chi}: \bar{V}_{D} \rightarrow V_{\kappa}$ be a function and $\chi_{\kappa}{ }^{\mathcal{K}}=\alpha_{\chi}\left(\left\{\chi_{h}, a_{h}\right\}\right), \chi_{\kappa}{ }^{\mathcal{K}}\left(\mathrm{X}_{h}\right)=\chi_{h}$, where $X_{h}$ is value of $\chi_{h}$ in a reference configuration.

\section{Definition 2:}

The deformation function associated with the distinguished family of subbodies $\mathcal{K}$ is a function $\chi_{\kappa}^{\mathcal{X}}$ which has the form $\chi_{\kappa}^{\mathcal{X}}=\alpha_{\chi}\left\{\left(\chi_{h}, a_{h}\right\}\right)$.

\section{Definition 3:}

The motion of the body $\mathcal{B}$ associated with the family of sets $\mathcal{K}$ is a continuous map $\chi_{i}:[0, T] \rightarrow\left\{\chi_{\kappa}^{\mathcal{K}_{3}}\right\}$.

Let us consider a function $\bar{T}: \mathcal{M}_{K} \rightarrow R^{P}, \bar{T}\left(\left\{K_{h}\right\}\right)=\left\{T_{h}\right\}$ which represents temperature. Let furthermore $I_{h}^{b} \subset I_{P}$ and $H_{T h}=\left\{T_{n}, n \in I_{h}^{b}\right\}$. Then, we introduce function $b_{h}$ by analogy to $a_{h}$ as $b_{h}:\left\{H_{T h}\right\} \rightarrow V_{b h}$ and $b:\left\{\left\{H_{T h}\right\}\right\} \rightarrow\left\{\left\{b_{h}\left(T_{n}\right)\right\}\right\}$.

Determination of the value $T_{h}$ is not so direct as defining $\chi_{h}$. In the last case we use geometrical interpretation. We cannot do this in the case of temperature. Discussed problem is connected with precise definition of the mapping $\pi_{T}$ considered as component of the dimensional reduction procedure. Such a definition allows one to distinguish the part of evolution of the system which is responsible for definition of temperature. 
Let $\bar{V}_{T M}=\left\{\left\{T_{h}, b_{h}\right\}, h \in I_{P}\right\}, V_{T M}=\{T(\mathbf{x}): \mathbf{x} \in \mathcal{X}(\mathcal{B})\}$. Let us consider a function $\alpha_{T}: \bar{V}_{T M} \rightarrow$ $V_{T M}$ and $T^{\mathscr{X}}=\alpha_{7}\left(\left\{T_{h}, b_{h}\right\}\right)$.

\section{Definition 4:}

The temperature field $T^{\mathcal{K}}$ associated with the distinguishedfamily of subbodies $\mathcal{K}$ is the field obtained with the help of the function $\alpha_{T}$ as $T^{\mathcal{X}}=\alpha_{T}\left(\left\{T_{h}, b_{h}\right\}\right)$.

We have obtained definition of the body, deformation function and motion of the body using an elementary dynamical system. The mappings $G_{v}, G_{x}$ determine connections between EDS and continuum description. Furthermore, temperature is connected with EDS by means of the mapping $\pi_{T^{*}}$

The spaces $\vec{V}_{D}$ and $\bar{V}_{T M}$ are finite dimensional. As a result we have obtained also finite-dimensional spaces $\alpha_{\chi}\left(\bar{V}_{D}\right)$ and $\alpha_{T}\left(\bar{V}_{T M}\right)$. This follows that finite-dimensional fields are considered on continuum only.

In order to introduce a continuum skeletal dynamical system we must have at our disposal balance of mass and energy equations also associated with the elementary dynamical system. Previously we have defined functions $\tilde{m}, J_{\varphi i j}, c_{\varphi}, E_{\varphi}, W_{\varphi i j}, R_{\varphi}$ which introduce masses, efflux of mass between subsystems, source of mass, energy, efflux of energy and source of energy related to subsystems respectively. These functions, indexed here by $\varphi$, are introduced in connection with the elementary dynamical system.

Let us consider the mapping $\tilde{m}: \mathcal{M}_{\Pi} \rightarrow\left\{\left\{m_{h}\right\}\right\}$ which determines a set of masses related to collection of dynamical systems. Let $\mathcal{M}_{M}=\left\{\left\{M_{h}\right\}\right\}$ and $M: \mathscr{M}_{K} \rightarrow \mathcal{M}_{M}$ be a mapping which assigns masses to each $K_{h}$. Masses $M_{h}$ related to continuum model are defined by means of the relation $M \circ G_{V}=i \circ \tilde{m}$, where $i$ is identity mapping. Thereby, a system of masses related to continuum is introduced by means of the mapping $\tilde{m}$ defined on elementary dynamical system.

We have defined body $\mathcal{B}$ associated with the elementary dynamical system. We define subbody also denoted by $\mathcal{B}=\bigcup_{h} K_{h}, h \in I_{B}$, where $I_{B} \subset I_{P}$ is an arbitrary subset of $I_{P}$. Let us introduce $M(\mathcal{B})=\sum_{h \in I_{B}} M_{h}$. Thereby, mass related to subbodies is defined as a kind of measure on the body.

The function $\mathscr{E}: \mathscr{M}_{K} \rightarrow\left\{\left\{\mathscr{S}_{h}\right\}\right\}$ determines distribution of energy on the family $\left\{K_{h}\right\}$ and $\mathscr{E}_{h}=\pi_{h} \circ \tilde{E}\left(\left\{K_{h}\right\}\right)$ assigned to each $K_{h}$ is defined by means of the relation $\mathscr{E} \circ G_{V}=i \circ E_{\varphi}$.

Source of mass $c_{\varphi}: \mathcal{M}_{\Pi} \rightarrow R^{P}$ and source of energy $R_{\varphi}: \mathcal{M}_{\Pi} \rightarrow R^{P}$ are defined now as $C: \mathscr{M}_{K} \rightarrow R^{P}, R: \mathcal{M}_{K} \rightarrow R^{P}$ by means of relations $C \circ G_{V}=i \circ \mathcal{C}_{\varphi}$ and $R \circ G_{V}=i \circ R_{\varphi}$. These quantities can be defined for subbodies with the help of formulas $\mathcal{E}(\mathcal{B})=\sum_{h} \mathcal{E}_{h}, C(\mathcal{B})=\sum_{h l} C_{h}$, $R(\mathcal{B})=\sum_{h} R_{h}$.

We assume without discussion of details that $\mathfrak{G}=E+\mathscr{T}$ is considered as a sum of internal energy and kinetic energy for continuum.

Interchange of mass and energy is described by efflux of mass, $J_{\varphi i j}: \mathscr{M}_{I I} \rightarrow R$ and efflux of energy $W_{\varphi i j}: \mathcal{M}_{I I} \rightarrow R$. These quantities are defined for continuum as $J_{i j}: K_{i} \times K_{j} \rightarrow R$, where 
$J_{i j}$ is determined by means of $J_{i j} \circ\left(G_{V i} \times G_{V j}\right)=i \circ J_{\varphi i j}$ and $W_{i j}: K_{i} \times K_{j} \rightarrow R$, where we obtain $W_{i j}$ fron $W_{i j} \circ\left(G_{V i} \times G_{V j}\right)=i \circ W_{\varphi i j}$.

Let us consider furthermore boundary of the body as $\delta \mathcal{B}=\delta \bigcup_{h} K_{h}$. We define $J(\delta \mathcal{B})$ $\sum_{i \in I_{B}, m \in I_{P}-I_{B}} J_{i m}$ and $W(\delta \mathscr{B})=\sum_{i \in I_{B}, m \in I P-I_{B}} W_{i m}$ as quantities referred to boundary of the body. Let $\delta \mathcal{B}_{s} \subset \delta \mathcal{B}$ be defined as $\delta \mathcal{B}_{s}=\bigcup_{h \in I_{s}}\left(\delta K_{h} \cap \delta \mathcal{B}\right), I_{s} \subset I_{B}$. We assume that pair of indexes $\{i, m\}$ is associated with $\delta \mathcal{B}_{s}$ if $\delta \mathcal{B}_{s}$ is a border between subsystems $i$ and $m$. Then, $J\left(\delta \mathcal{B}_{s}\right)=\sum_{i, m} J_{i m}$.

Using terms of continuum associated with the elementary dynamical system introduced above we can express balance of mass equation for continuum which corresponds to (3) as

$$
\dot{M}(\mathscr{B})+J(\delta \mathcal{B})-C(\mathcal{B})=0
$$

with the additional condition as a counterpart of (4) and satisfied for arbitrary $\delta \mathcal{B}_{s} \subset \delta \mathcal{B}$

$$
J\left(\delta \mathcal{B}_{s}\right)=\bar{J}\left(\delta \mathscr{B}_{s}\right)
$$

for a given $\bar{J}\left(\delta \mathcal{B}_{s}\right)$.

The balance of energy equation corresponding to (5) and now expressed in terms of continuum is assumed in the following form

$$
\dot{E}(\mathcal{B})+\mathscr{T}(\mathcal{B})+W(\delta \mathcal{B})-R(\mathcal{B})=0
$$

with the additional condition corresponding to (6) and satisfied for arbitrary subboundary $\delta \mathcal{B}_{s} \subset \delta \mathcal{B}$ of the body $\mathcal{B}$

$$
W\left(\delta \mathcal{B}_{\mathrm{s}}\right)=\bar{W}\left(\delta \mathcal{B}_{\mathrm{s}}\right)
$$

for a given $\vec{W}\left(\delta \mathcal{B}_{\mathrm{s}}\right)$.

Introduced here equations of balance of mass and energy are the first stage for postulation the skeletal dynamical system. In the next step we assume representations of quantities introduced in (7-10). They are parameterized by some constants which have to be identified. Identification of these constants is realized with the help of solutions of the elementary dynamical system.

Continuum fields on the body are introduced by means of previously determined discrete fields with the help of mappings similar to $\alpha_{x}$ and $\alpha_{T}$ for deformation and temperature. They should fulfill well known integral relations between densities and discrete values of quantities on each subbody. Consequently, discussed here continuum mechanics is characterized by finite-dimensional spaces of fields. In classical case such spaces are infinite-dimensional. This difference is important. Derived equations are finite-dimensional and we do not apply discretization methods. In discussed case a continuum model with finite-dimensional fields is inherently connected with determination of degree of averaging what is expressed by option of sets $K_{h}$ and the skeletal dynamical system. As a result we introduce by this, formalization of scale of averaging. Such a fact is important for description of processes associated with phenomena relating to several scales. Thereby this is also important for biological systems. 
Let us assume that the elementary dynamical system is determined by equations of molecular dynamics. Then, integration of the molecular dynamics with continuum mechanics consists in fact that we use continuum skeletal dynamical system in the framework of the dimensional reduction procedure.

Let us note that connections of molecular dynamics and continuum mechanics are discussed in literature. Such considerations are carried out usually in the context of statistical mechanical calculations supported by molecular dynamics [7, 8], Considerable achievement for cooperation of both theories is given by Prrinello and Rahman in [9].

\subsection{Final comments}

We have introduced collection of dynamical systems with dimensional reduction as a multiscale method of modelling. Final aim of this approach is to provide the reduced dynamical system which describes our object in an averaged way.

In order to realize the dimensional reduction procedure we have to divide EDS into subsystems, determine mappings $\pi_{T}, \pi_{\mathrm{fT}}$ and postulate form of $S D S$. Let us notice that $S D S$ can be arbitrary in fact. Indeed, we can identify constants of $R D S$ by means of some general procedures. An example of such a procedure is discussed in (44). However, then the main question is related to quality of approximation. In order to improve the quality of approximation we should introduce into form of SDS additional information.

We discuss in Subsection 2.2 balance of mass and energy equations for collection of dynamical systems. Let us notice that such equations are not necessary in general. We discuss them just in order to provide additional information into form of SDS. When the structure of SDS is in accordance with the balance of mass and energy equations then we can expect that such laws are well approximated by the reduced dynamical system.

We can consider two extreme points of view on methods of postulating of SDS. The first one is related to situation when we postulate $S D S$ arbitrarily, neglecting accessible information from $E D S$. In such a case we can expect that approximation is not perfect. The second one corresponds to situation when we derive form of $S D S$ formally from equations of $E D S$. This way can be complicated for complex systems. Therefore, in practice, we expect an intermediate situation. We postulate $S D S$ by means of some additional information obtained from $E D S$, perhaps by partial derivation of some results. Just such a situation takes place when we derive general form of balance of mass and energy equations for collection of dynamical systems.

In particular, we can also derive balance of mass and energy equations assuming that SDS represents continuum skeletal dynamical system. Then, we introduce additional information connected with structure of continuum. We can consider the temperature field for instance. It means that we describe evolution of our object with considerable degree of averaging. When we discuss an averaged model of single molecule we usually do not use continuum description. Then, we introduce variables which describe dynamics of small scale in less averaged way. 


\section{DEFINITIONS OF THE MOLECULAR RECOGNITION AND INTEGRITY PROPERTY}

Discussed above collection of dynamical systems with dimensional reduction provides a mathematical framework for unification of various theoretical methods within one multiscale description. Such a mathematical formalism should be supplemented by a set of notions which correspond to characteristic properties of biological structures. In this section we define necessaiy conditions corresponding to precise realization of a selected chemical reaction as well as for integrity of a system of chemically reacting molecules.

We define first the molecular recognition. The molecular recognition is widely applied within supramolecular chemistry [10] by scientists which realize in practice synthesis of large molecules.

Following the papers of Lehn [10] we find that "molecular recognition has been defined as a process involving both binding and selection of substrate(s) by a given receptor molecule, as well as possibly a specific function".

A mathematical definition of the molecular recognition has been introduced in [11]. This notion is viewed to be important for characterization of biological structures. Therefore, we introduce here this definition.

Let us consider two molecules $a$ and $b$ and the reaction $a+b \rightarrow a b$ denoted by $R$. Let $\mathcal{V}_{C}=\left\{\mathbf{R}_{i}\right\}$ be configuration space for the discussed molecular system and $V: \mathcal{V}_{C} \rightarrow R$ be the potential energy. Then, $\mathbf{v}_{\boldsymbol{i}}=\dot{\mathbf{R}}_{\boldsymbol{i}}$ and $m$. are velocity and mass of z'-th particle. Realization of the reaction can be expressed by evolution of configuration of the whole system. Final state of this system is described by evolution in a catchment region $C_{R}$ corresponding to the reaction $R$. Catchment regions for the potential energy are defined in $[14,15]$ as equivalence classes for points belonging to $\mathcal{V}_{C}$ which have steepest descent paths with ends at the same minimum.

We introduce the following equations describing evolution of our molecular system in solution [12]

$$
\begin{gathered}
\dot{\mathbf{R}}_{i}=\mathbf{v}_{i}, \\
m_{i} \dot{\mathbf{v}}_{i}=-c_{i} \mathbf{v}_{i}-\frac{\partial V}{\partial \mathbf{R}_{i}}+\mathrm{F}_{S i}+\mathbf{f}_{i},
\end{gathered}
$$

where $\mathrm{c}_{i}$ are friction constants, $\mathrm{c}_{i} \mathbf{v}_{i}$ represent friction forces induced by solvent, $\mathrm{F}_{S i}$ are stochastic forces following from solvent-molecule interactions and $f_{i}$ are external forces acting on the system.

We consider equations (11) as the starting point for introduction of definition of the molecular recognition.

Let $\mathbf{D}=\{\mathbf{R}, \mathbf{v}\} \in \mathcal{V}_{D}^{\prime}$ represent variables of our equations, where $\mathcal{V}_{D}$ stands for corresponding phase space. We introduce $\pi_{C}: \mathcal{V}_{D} \rightarrow \mathcal{V}_{C}$ as projection of the phase space into the configurational space. Let $\mathbf{D}\left(\mathbf{D}_{0}, \mathcal{L}(t)\right)$ be a solution of our equations with an initial condition $\mathbf{D}_{0}=\mathbf{D}\left(t_{0}\right)$ which represents initial state of molecules $a$ and $b$. Since forces $\mathcal{L}(t)=\left\{\mathbf{F}_{S i}\right\}$ are considered to be a stochastic process, then $\mathbf{D}(t)$ is also a realization of a stochastic process. 


\section{Definition 5}

We say that two molecules $a$ and $b$ undergo the molecular recognition with respect to the reaction $\mathscr{R}$, with probability $p$, if they find themselves in a state $\mathbf{D}_{0} \in U_{S} . U_{S} \subset \mathcal{V}_{D}$ is a maximal set called the range of selection, which satisfies the condition that there exists $t_{k}$ such that for each $\mathbf{D}_{0} \in U_{S}, \mathscr{P}\left(\left\{\pi_{C}\left(\mathbf{D}\left(\mathbf{D}_{0}, \mathcal{L}(t)\right)(t)\right) \in C_{R}, t>t_{k}\right\}\right)=p \leq 1$, and $\mathcal{P}$ is a probability measure related to this problem.

Introduced above definition fulfills expectations related to the Lehn formulation. If the molecules $a$ and $b$ are in state $\mathbf{D}_{0} \in U_{S}$ then they have to react in order to find themselves in the final binding state in case of $p=1$. Form of the set $U_{s}$ represents information contained in the system which characterizes conditions leading to realization of this reaction. Thereby, the set $U_{s}$ is responsible for selection of substrates.

This definition suggests that process of bonding of two atoms is also associated with molecular recognition. Indeed, this is the case. However, the range of selection is then rather simple and not too interesting. In the case of molecular reactions determination of the range of selection set seems to be very difficult in general. In particular, it depends on model of solvent applied in calculations. Summarizing, the definition of the molecular recognition indicates that knowledge of range of selection set is a necessary condition for realization of precise reactions in molecular systems and, in particular, in biological molecular systems. Therefore, a consequence of this definition is a necessity of development the part of chemical reaction dynamics which is directed especially towards determination of the range of selection.

We observe that chemical reactions in living organisms are highly organized and create an integrated system. The definition of the molecular recognition allows one to formulate integrity property for a chain of chemical reactions.

We define a chain of chemical reactions $\left\{C R_{\alpha}\right\}$ by means of the formula

$$
a_{\alpha}+b_{\alpha} \rightarrow a_{\alpha+1}+\left\{d_{\alpha i}\right\}, \quad \alpha \in I_{C R}
$$

Thus, the characteristic property of the chain of reactions consists in fact that the product $a_{\alpha+1}$ of the reaction $C R_{\alpha}$ is a substrate for the next reaction $C R_{\alpha+x^{*}}$. The substrate $b_{\alpha+x}$ is taken from an environment.

Values of positions and velocities of nuclei of atoms belonging to molecules $a_{\alpha}$ and $b_{\alpha}$ at the time instant $t$ are denoted by $\{\mathbf{R}, \mathbf{v})\left\{\left\{a_{a}, b_{\alpha}\right\}\right)(t)$. Let $U_{\alpha}$ stand for range of selection for the reaction $C R_{\alpha^{*}}$. Let us consider a time interval $\Delta_{\alpha}=\left[t_{p \alpha}, t_{k \alpha}\right]$ connected with a stage of realization of the chain of reactions. We assume that an instant for inception of the reaction $C R_{\alpha}$ belongs to $\Delta_{a}$.

\section{Definition 6}

We say that the chain of the chemical reactions $\left\{C R_{\alpha}\right\}, \alpha \in I_{C R}$ has the integrity property if $\{\mathbf{R}, \mathbf{v}\}\left(\left\{a_{\alpha}, b_{\alpha}\right\}\right)(t) \in U_{\alpha}$ for some $t \in \Delta_{\alpha}$ for each $\alpha \in I_{C R}$. 
The range of selection is of fundamental importance for this definition. Each step of realization of the chain of reactions has to be preluded by molecular recognition. This has to be interpreted as a necessary condition for precise realization of this system of chemical reactions.

Summarizing, the mathematical definition of the molecular recognition enables us to discuss problem of integrity of larger system of chemical reactions. This property also distinguishes biological systems from other ones.

\section{DEFINITION OF THE MOLECULAR REACTIVE STRUCTURE}

Considerable part of molecules in cell does not change their chemical composition. However, such molecules can undergo structural transformations and take part in chemical reactions. Thus, they are similar to catalysts. However, category of catalysts is functionally directed to controlling chemical reactions. We would like to define molecular reactive structures as all stable with respect to composition molecules which can take part in chemical reactions but their function can be various. In particular they can be enzymes or they can create larger, solids like structures.

We formulate definition of the molecular reactive structure considering molecule as a set of material points corresponding to nuclei of atoms. We introduce notation $S(\mathbf{R})$ for a system of the material points representing molecule and $\mathbf{R}$ for its current configuration. We introduce notation from the set theory for systems like $S$. Thus, two subsystems $S_{1} \cup S_{2}=S$ create a new molecule represented by $S$.

Let $\mathbf{R}_{R S}=\left\{\mathbf{R}_{i}\right\}, i \in I_{R S}$ stand for positions of material points within the reactive structure $S_{R S}$. The configuration $\mathbf{R}_{R S}$ can undergo transition between catchment regions during evolution. We admit, in general, transitions for the molecular reactive structure which do not destroy an admissible structure of interatomic bonds.

Let us introduce a relation $\mathfrak{B}_{\alpha} \subset I_{R S} \times I_{R S}$ which consists of pairs of nuclei which are joined by interatomic bond $b\left(\mathbf{R}_{i}, \mathbf{R}_{j}\right)$. Existence of such bonds can be determined by means of methods of quantum chemistry [13].

Let $\mathcal{A}_{B}$ be a set of all relations $\mathcal{B}_{\alpha}$ which represents an admissible structure of interatomic bonds within $S_{R S}$. The term "admissible" corresponds to current requirements related to described structure. Thus, we can accept the case when the set $\mathcal{A}_{B}$ consists of one element only. Then, all interatomic bonds cannot be changed with respect to the relation classifying them. However, we can accept the situation when a reorganization of interatomic bonds structure, without unwanted changes, happens.

With the help of the set $\mathcal{A}_{B}$ we introduce a relation between relations. Thus, $\mathfrak{B}_{\alpha} \equiv \mathscr{A} \mathfrak{B}_{\alpha^{\prime}}$, when $\mathfrak{B}_{\alpha}, \mathcal{B}_{\alpha^{\prime}} \in \mathcal{A}_{B}$.

Domain $\mathcal{V}_{C}$ of the potential energy can be divided into catchment regions $C_{a}$ connected with minima $\boldsymbol{c}_{\alpha}$ of $V$ as $\mathcal{V}_{C}=\cup_{\alpha} C_{\alpha}[14,15]$. If the molecule $S_{R S}$ interacts with other particles during a chemical reaction then the configuration space is extended. As a result the potential energy $V=V\left(\mathbf{R}_{R S}, \mathbf{R}_{E}\right)$ depends on additional variables $\mathbf{R}_{E}$ representing a system of external molecules $S_{E}$. Decomposition of $\mathcal{V}_{C}$ into catchment regions depends now on $\mathbf{R}_{E}$ and we have $C_{\alpha}=\operatorname{C} \alpha\left(\mathbf{R}_{E}\right)$. 
Let us assume that $\left\{\mathbf{R}_{R S}, \mathbf{R}_{E}\right\}(t)$ represents process of a chemical reaction. At an initial instant $t_{I}$ the molecules $S_{R S}$ and $S_{E}$ are separated. At a final instant $t_{F}$ they are also separated. This can be expressed by fact that $\left.V\left(\mathbf{R}_{R S}, \mathbf{R}_{E}\right)\right|_{t_{\text {, }} t_{F}}=V_{R S}\left(\mathbf{R}_{R S}\right)+V_{E}\left(\mathbf{R}_{E}\right)$ in a neighbourhood of initial and final positions of this molecular system. We introduce a set of admissible processes $\mathbf{R}_{E}(t) \in \mathcal{A}_{R S}$ satisfying discussed above conditions with respect to $S_{R S}$ and which ensures realization of this reaction process.

\section{Definition 7}

We call a molecule $S_{\alpha}$ the molecular reactive structure if configurations $\mathbf{R}_{R S}$ induced by all admissible processes $\mathbf{R}_{E}(t) \in \mathcal{A}_{R S}$ are contained within domain $\mathcal{V}_{C}=\bigcup_{a \in I_{C}} C_{\alpha}\left(\mathbf{R}_{E}(t)\right)$ between initial andfinal instants $t_{I}$ and $t_{F}$. Furthermore, then we also have $\mathfrak{B}_{\alpha} \equiv \mathcal{A B}_{\alpha}$ for each pair $\alpha, \alpha^{\prime} \in I_{C}$ which appear during evolution of the configuration $\mathbf{R}_{R S}$ through various catchment regions.

This definition expresses fact that the molecular reactive structure takes part in a chemical reaction but interatomic bonds can undergo evolution without destroying their admissible structure only. Thereby, deformation and structural transformations of the molecule $S_{R S}$ are admissible.

\section{CONCEPT OF MECHANICS OF REACTIVE NANOSTRUCTURES}

\subsection{Skeletal dynamical system for reactive nanostructures}

We have introduced molecular reactive structures using atomic level of description. However, we can discuss reactive structures also by more averaged descriptions.

Let us consider behaviour of actin cytoskeleton [16,1], This molecular structure undergoes considerable reorganization in order to realize motility of cell. In particular cytogel into cytosol transformation is realized by destruction of a meshwork of actin filaments. Assembly of contractile actin-myosin filaments is organized within cell for generation active stress. Such a medium can be described by means of continuum models [1] in a very averaged way. Then, the problem is to postulate form of constitutive equations taking into account high controllability of this medium.

Elementary mechanisms of reorganization of the actin cytoskeleton are related to single molecules. Actin filaments are assembled from monomers [16], Protein molecules take part in regulation of these processes $[1,17,18]$

Above concise discussion suggests that considered here processes are related to single molecules or to a system of such molecules which can undergo a reorganization. Thus, evolution of this system depends on properties of single molecules as well as on processes responsible for the reorganization. Modelling of single molecules considering all atoms is too complicated for description of the multimolecular processes. Therefore, it seems to be the most appropriate to elaborate models of single molecules which describe evolution of groups of atoms within them 
in an averaged way. Then, we have to use a nanoscale level of averaging properties of intramolecular processes. Furthermore, we should be able to describe mentioned above processes of reorganization as well as interactions with external chemical reactions.

We come across in literature some models which consider reduced number of degrees of freedom for describing evolution of molecules. Let us mention for instance the paper [19].

We tend towards construction of a skeletal dynamical system for our reactive nanostructures. To this end we should distinguish first a set of new variables related to the reduced level of description.

In many cases, during modelling, we are able to distinguish slowly and quickly varying processes realized within considered system. We assume that this is the case for evolution of our molecule modelled. Thus we consider thermal vibrations as quickly varying processes and structural transformation or a deformation of the molecule as the slowly varying one. Such division of processes we call $S Q$-decomposition. We introduce variables which reflect this property, by means of a set of mappings defined to this end.

We consider, as previously, a molecule $S\left(\mathbf{R}_{R S}\right)$ modelled by means of a set of material points. We distinguish subsystems within such molecule as $S\left(\mathbf{R}_{R S}\right)=\bigcup_{h \in I_{A}} S_{h}\left(\left\{\mathbf{R}_{h j}\right\}\right)$ composed of determined groups of atoms.

Let us note that many small molecules appear in biological systems repeatedly, what gives a hope to obtain a degree of universality in division into subsystems.

We introduce three kinds of variables on the dimensionally reduced level of description. The first one represents configurational variables $\boldsymbol{Q}_{h}=\left\{Q_{h p}\right\}$ and velocities $\mathbf{w}_{h}=\dot{\mathbf{Q}}_{h}$, which approximately describe evolution of the whole group of particles within $h$-th subsystem. They describe slowly varying processes. We introduce also the configuration space $\overline{\mathcal{V}}_{C}=\{\mathbf{Q}\}=\left\{\left\{\mathbf{Q}_{h}\right\}\right\}$ and the corresponding phase space $\overline{\mathcal{V}}_{D}=\left\{\left\{\mathbf{Q}_{h}, \mathbf{w}_{h}\right\}\right\}$.

The second kind of variables $\theta_{h}$ describes in an averaged way quickly varying processes and is related directly to balance of energy equation. The third group of variables $\eta_{h}$ is related to quickly vaiying processes and needs postulating additional evolution equations.

Let us introduce spaces of processes $\mathcal{V}_{T}=\left\{\left\{\mathbf{R}_{i}, \mathbf{v}_{i}\right\}(t): i \in I_{R S}, t \in T\right\}$ related to atomic level of description and $\overline{\mathcal{V}}_{D T}=\left\{\left\{\mathbf{Q}_{h}, \mathbf{w}_{h}\right\}(t): h \in I_{A}, t \in T\right\}, \overline{\mathcal{V}}_{\theta T}=\left\{\left\{\theta_{h}\right\}(t): h \in I_{A}, t \in T\right\}$, $\overline{\mathcal{V}}_{\eta T}=\left\{\left\{\eta_{h}\right\}(t): h \in I_{A}, t \in T\right\}$ and $\overline{\mathcal{V}}_{T}=\overline{\mathcal{V}}_{D T} \times \overline{\mathcal{V}}_{\theta T} \times \overline{\mathcal{V}}_{\eta T}$, where ris a time interval.

New variables are obtained by means of the mappings $\pi_{T}: \mathcal{V}_{T} \rightarrow \overline{\mathcal{V}}_{T}, \pi_{T}=\pi_{S T} \times \pi_{Q T}$ and $\pi_{S T}: \mathcal{V}_{T} \rightarrow \overline{\mathcal{V}}_{D T}, \pi_{Q T}: \mathcal{V}_{T \rightarrow} \rightarrow \overline{\mathcal{V}}_{\theta T} \times \overline{\mathcal{V}}_{\eta T}$

Structure of the mappings $\pi_{S T}$ and $\pi_{Q T}$ is obtained by using decomposition of variables. In order to illustrate this let us take a set of time instants $t_{0} \quad\left\langle t_{1}<\ldots \quad\left\langle t_{K}\right.\right.$ which belong to the time interval $T=\left[t_{0}, t_{K}\right], t_{K}=t_{0}+T$, and let $I_{K}=\{0,1, \ldots, K\}$. By means of these instants we divide the time interval into the sum $T=\mathrm{U}_{k} T_{k}, T_{k}\left[t_{k-1}, t_{k}\right], \quad k=1, \ldots, K$. Then, for each $k$ we can calculate the value of $\widetilde{\mathbf{R}}_{k}$ as

$$
\tilde{\mathbf{R}}_{k}=\frac{1}{T_{K}} \int_{T_{k}} \mathbf{R}(t) d t
$$


A value $\tilde{\mathbb{R}}_{0}=\mathbf{R}\left(t_{0}\right)$ is assigned to $k=0$. With the aid of sequence of values $\left\{\tilde{\mathbf{R}}_{k}\right\}, k \in \bar{I}_{K}$ we can generate a function $\tilde{\mathbf{R}}(t)=\mathscr{I}_{R}\left(\left\{\tilde{\mathbf{R}}_{k}\right\}\right)$, where $\mathscr{g}_{R}$ is an approximation procedure. Now, we are able to decompose the variable $\mathbf{R}(t)$ into two summands

$$
(t)=\tilde{\mathbf{R}}(t)+\delta \mathbf{R}(t)
$$

Thus, $\tilde{\mathbf{R}}(t)$ represents the slowly varying part of $\mathbf{R}(t)$ and $\delta \mathbf{R}(t)$ its quickly varying part.

We assume that the mapping $\mathscr{S}_{R}$ satisfy the condition $\int_{T_{k}}(\mathbf{R}-\tilde{\mathbf{R}}) d t=\int_{T_{k}} \delta \mathbf{R} d t \approx 0$, where „ $\approx$ " means equality with an admissible error. In general methods of determining of $\mathscr{g}_{R}$ are an open problem.

Let $A_{t}(\{\mathbf{R}, \dot{\mathbf{R}}\}(t))=\{\tilde{\mathbf{R}}, \delta \mathbf{R}, \dot{\widetilde{R}}, \delta \dot{\mathbf{R}}\}(t)$ be mapping which realizes $S Q$-decomposition. We assume futher that there exists mapping $\pi_{s}$ and $\pi_{0}$ which give direct assignation of new reduced variables to those obtained by means of $A_{t}$. We would say that $\pi_{s}$ and $\pi_{0}$ takes into account also a space averaging imposed on previously obtained time averaging introduced by $A_{t}$. As a result we obtain

$$
\pi_{T}=\left\{\pi_{S T}, \pi_{Q T}\right\}=\left\{\pi_{S} \circ A_{\ell}, \pi_{Q} \circ A_{\ell}\right\}
$$

Similar $S Q$-decomposition can be carried out for forces $\mathbf{f}$. To this end we calculate the following time averaged quantities

$$
\tilde{\mathbf{f}}_{k}=\frac{1}{T_{k}} \int_{T_{k}} \mathbf{f}(t) d t .
$$

Then, we have $\tilde{\mathbf{f}}(t)=\mathscr{g}_{f}\left(\tilde{\mathbf{f}}_{k}\right)$ with the help of an approximation procedure $\mathscr{g}_{f}$. Finally, we obtain the decomposition

$$
\mathbf{f}(t)=\tilde{\mathbf{f}}(t)+\delta \mathbf{f}(t)
$$

Let $B,(f(t))=\tilde{\mathbf{f}}(t), \delta f(t)\}$ and $\pi_{S f} \circ B_{l}(\mathbf{f}(t))=\tilde{\mathbf{f}}_{S}, \pi_{Q f} \circ B_{l}(\mathbf{f}(t))=\tilde{\mathbf{f}}_{Q}$. Thereby, we obtain the general form of the map $\pi_{f T}$ by

$$
\pi_{f T}=\left\{\pi_{S f} \circ B_{t}, \pi_{Q f} \circ B_{t}\right\}
$$

and $\pi_{f T}(\mathbf{f}(t))=\left\{\tilde{\mathbf{f}}_{S}, \tilde{\mathbf{f}}_{Q}\right\}(t)$

In general it is expected that mappings $\pi_{T}$ and $\pi_{f T}$ are not entirely independent. We postulate that there exist relation $\mathscr{R}_{L}\left(\pi_{l}, \pi_{f T}\right)$ which join considered two mappings. In discussed here case we assume that $\mathscr{R}_{L}=I$ or in other words, the same procedures are represented by $\pi_{T}$ and $\pi_{f T}$.

Discussed above $S Q$-decomposition is similar to some degree to the Parinello Rahman method applied in molecular dynamics [9], 
In order to construct a skeletal dynamical system we have to postulate form of total energy $\mathscr{E}$ of the system modelled. We assume that

$$
\mathscr{E}=\sum_{h} E_{h}=\sum_{h}\left(V_{h}+\widetilde{T_{h}}\right)
$$

where $\mathcal{T}^{\prime}=\sum_{h} \mathcal{T}_{h}$ is kinetic energy corresponding to slowly varying processes and

$$
\sum_{h} V_{h}=\sum_{h}\left(V_{S h}+V_{Q h}\right)=\Phi+\mathscr{E}_{Q}
$$

is decomposed into parts related to slowly and quickly varying processes. Furthermore, we assume that

$$
\begin{aligned}
& V_{S h}=C_{S h \gamma} \Psi_{S h \gamma}\left(\mathbf{Q}_{h}, a_{Q h}\right), \\
& V_{Q h}=C_{Q h \delta} \Psi_{Q h \delta}\left(\theta_{h}, a_{\theta h}\right),
\end{aligned}
$$

where $a_{Q h}$ and $a_{\theta h}$ are functions of kinematical dependence between subsystems related to $\mathbf{Q}_{h}$ and $\theta_{h}$ correspondingly. They are defined in similar way as this is done in Subsection 2.3 for deformation function and temperature. We admit dependence $C_{S h r}, C_{Q h \delta}$ on $\mathbf{Q}_{h}, \theta_{h}, \quad \eta_{h}$ in the sequel.

Let us consider a given values of variables $\{\theta, \eta\}$. Then, the function $\tilde{\Phi}=\Phi(\{\theta, \eta\})(\mathbf{Q})$ defined in (20) depends on configurational variables only. We also assume that $\overrightarrow{\mathbf{Q}}=\left\{\overline{\mathbf{Q}}_{h}\right\}$ stands for minimum of this function.

Let $A_{R}: \overline{\mathcal{V}}_{C} \rightarrow \overline{\mathcal{V}}_{C}, T: \overline{\mathcal{V}}_{C} \rightarrow \overline{\mathcal{V}}_{C}$ be mappings which realize the same rotation and translation for each component of $\mathrm{Q}$ belonging to the configuration space $\overline{\mathcal{V}}_{C}$. Then the transformation

$$
\mathbf{Q}=A_{R}(\overline{\mathbf{Q}})+T(\overline{\mathbf{Q}})
$$

represents rigid transformation of the configuration $\overline{\mathbf{Q}}$. In other words we have defined rigid motion of the molecule $S$ on the dimensionally reduced level.

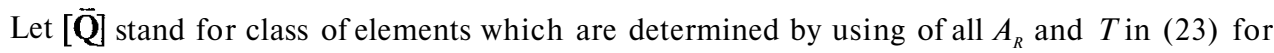
given $\overline{\mathbf{Q}}$ and $\mathscr{M}$ be set of all manifolds in $\overline{\mathcal{V}}_{C}$. We introduce a set of representatives of the equivalence classes in the form

$$
H=\left\{\mathbf{Q}: \mathbf{Q}, \mathbf{Q}_{1} \in H \Rightarrow[\mathbf{Q}] \neq\left[\mathbf{Q}_{1}\right], \quad H \in \mathscr{M}\right\}
$$

The set $H$ represents deformations of the molecule $S$ detennined on the dimensionally reduced level of description. $H$ is viewed to be manifold since deformation of the molecule is considered as a continuous process. 
The domain of the function $\widetilde{\Phi}$ can be expressed as a fiber bundle [20]

$$
\overrightarrow{\mathcal{V}}_{C}=H \times_{f} F_{R}(\mathbf{Q}), \quad \mathbf{Q} \in H
$$

where $\mathrm{x}_{f}$ stands for symbol of generalized Cartesian product of $H$ and a chosen fiber $F_{R}(\mathbf{Q})$ identified with the equivalence class $[\mathbf{Q}], H$ is considered as basis of the fiber bundle.

We assume that the function $\Phi$ has the following properties for each given values of $\{\theta, \eta\}$

$$
\Phi(\{\theta, \eta\})(\mathbf{Q})=\Phi(\{\theta, \eta\})\left(\mathbf{Q}_{1}\right)
$$

for all $\mathbf{Q}, \mathbf{Q}_{1}$ belonging to the same $F_{R}$ and this property is the case for all $F_{R}$. In other words the function $\Phi$ does not change its value during a rigid motion.

Let $\left[0, s^{*}\right] \subset R$ be an interval. The steepest descent path $p(\mathbf{Q}, \cdot):\left[0, s^{*}\right] \rightarrow \overline{\mathcal{V}}_{C}$ with the origin $\mathbf{Q}$ is defined as a path tangent to the vector $g=-\partial \widetilde{\Phi} / \partial \mathbf{Q}$. Then, we assume also that $p(\mathbf{Q}, 0)=\mathbf{Q}$

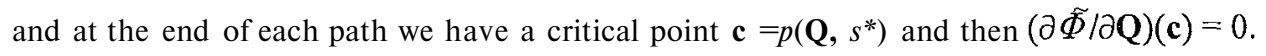

Two points $\mathbf{Q}, \mathbf{Q}_{1}$ are called equivalent if $p\left(\mathbf{Q}, s^{*}\right)=p\left(\mathbf{Q}_{1}, s^{*}\right)$. In other words equivalent points are origins of steepest descent paths having the same ends as a critical point.

\section{Definition 8:}

Equivalence classes of the relation defined by the steepest descent path are called catchment regions associated with the function $\tilde{\Phi}$.

As a result of defining the equivalence relation in $\overline{\mathcal{V}}_{C}$ we obtain decomposition of the domain of the function $\widetilde{\Phi}$ as follows

$$
\overline{\mathcal{V}}_{C}=\bigcup_{\alpha} C_{\alpha}(\{\theta, \eta\})
$$

where $C_{\alpha}$ are the catchment regions.

The catchment regions depend on $\{\theta, \eta\}$ since the function $\widetilde{\Phi}$ is defined for given $\{\theta, \eta\}$. Thus, evolution of these variables can lead to qualitative changes of the catchment region division given in (27).

The kinetic energy term $\mathcal{T}=\mathcal{T}\left(\mathbf{w}_{h}, \dot{a}_{Q h}\right)$ depends on slowly varying variables only and inertia characteristics. Let us calculate $\dot{\mathcal{T}}_{h}$. To this end we assume that the function $\mathscr{T}_{h}$ has properties

$$
\frac{\partial \mathscr{T}_{h}}{\partial w_{h p}} \approx \frac{\partial^{2} \mathcal{T}_{h}}{\partial w_{h p} \partial w_{h q}}(0) w_{h q}, \quad \frac{\partial \mathcal{T}_{h}}{\partial \dot{a}_{\underline{Q} h}} \approx \frac{\partial^{2} \mathscr{T}_{h}}{\partial \dot{a}_{\underline{Q} h}^{2}}(0) \dot{a}_{Q h}
$$

expressed by means of the Taylor expansion. Finally we obtain 


$$
\begin{aligned}
& \dot{\mathcal{T}}_{h}=\frac{\partial^{2} \mathcal{T}_{h}}{\partial w_{h p} \partial w_{h q}}(0) w_{h q} \dot{w}_{h p}+\frac{\partial^{2} \mathcal{T}_{h}}{\partial \dot{a}_{Q h}^{2}}(0) \frac{\partial \dot{a}_{Q h}}{\partial \dot{Q}_{j p}} \frac{\partial \ddot{a}_{Q h}}{\partial \ddot{Q}_{k q}} \dot{Q}_{j p} \ddot{Q}_{k q}= \\
& =M_{h p q} w_{h q} \dot{w}_{h p}+I_{h j q k r} \dot{Q}_{j q} \ddot{Q}_{k r}=\left(M_{h p q} \dot{w}_{h p}\right) \dot{Q}_{h q}+\left(I_{h j q k} \ddot{Q}_{k r}\right) \dot{Q}_{j q}
\end{aligned}
$$

We use usual summation by means of the symbol $\Sigma$ for indexes of subsystems $h \in I_{G}$ and $m \in I_{O}$ which are related to main division into subsystems. For the remaining indexes the summation convention is applied.

Taking into account $S Q$-decomposition we modify the general balance of energy equation (5) into the form

$$
\sum_{i \in I_{G}}\left(\dot{E}_{i}-R_{S i}-R_{Q i}+\sum_{i \in I_{O}}\left(W_{S i j}+W_{Q i j}\right)\right)=0
$$

Thereby, sources of energy considered within subsystems and efflux of energy are decomposed into two parts.

We assume that $C_{S h \gamma}$ and $C_{Q h \delta}$ are constant at this stage of derivation. Furthermore we postulate particular forms of $R_{s h}=\bar{f}_{S h q} \dot{Q}_{h q}$ and $W_{S h m}=-\bar{f}_{S h m q} \dot{Q}_{m q}$. Then, taking into account (19)-(22) and (28), the balance of energy (29) is now expressed by means of the formula

$$
\begin{aligned}
& \sum_{h \in I_{G}}\left\{\left[C_{S h \mu} \frac{\partial \Psi_{S h \mu}}{\partial Q_{h q}} \dot{Q}_{h q}+C_{S h \mu} \frac{\partial \Psi_{S h \mu}}{\partial a_{Q h}} \frac{\partial a_{Q h}}{\partial Q_{j s}} \dot{Q}_{j s}+C_{Q h \nu} \frac{\partial \Psi_{Q h \nu}^{\prime}}{\partial \theta_{h}} \dot{\theta}_{h}+\right.\right. \\
& \left.+C_{Q h \nu} \frac{\partial \Psi_{Q h \nu}}{\partial a_{\theta h}} \frac{\partial a_{\theta h}}{\partial \theta_{j}} \dot{\theta}_{j}\right]+\left(M_{h p q} \dot{w}_{h p}\right) \dot{Q}_{h q}+ \\
& \left.+\left(I_{h j s k r} \dot{Q}_{k r}\right) \dot{Q}_{j s}-\bar{f}_{S h q} \dot{Q}_{h q}-R_{Q h}+\sum_{m \in I_{o}}\left(-\bar{f}_{S h m q} \dot{Q}_{m q}+W_{Q h m}\right)\right\}=0
\end{aligned}
$$

with additional conditions corresponding to (6)

$$
W_{Q h m}=\bar{W}_{Q h m}, \quad h \in I_{G}, \quad m \in I_{O} .
$$

Owing to particular form of $W_{S h m}$ assumed, an additional condition for this quantity appears and will be discussed below.

At this moment we admit again dependence of $C_{s h y}, C_{Q h v}$ on previously considered variables. We assume that terms $\dot{C}_{S h \mu} \Phi_{S h \mu} \neq 0$ and $\dot{C}_{Q h v} \Phi_{Q h \nu} \neq 0$. We take into account these terms in the balance of energy equation. Consequently, next transformed version of (30) is given by 


$$
\begin{aligned}
& \sum_{h \in I_{G}}\left\{\left[C_{S h \mu} \frac{\partial \Psi_{S h \mu}}{\partial Q_{h q}}+\sum_{h^{\prime}} C_{S h^{\prime} \mu} \frac{\partial \Psi_{S h^{\prime} \mu}}{\partial a_{Q h^{\prime}}} \frac{\partial a_{Q h^{\prime}}}{\partial Q_{h q}}+M_{h p q} \dot{w}_{h p}+\sum_{h^{\prime}} I_{h^{\prime} h q k r} \ddot{Q}_{k r}-\bar{f}_{S h q}\right] \dot{Q}_{h q}+\right. \\
& +C_{Q h \nu} \frac{\partial \Psi_{Q h v}}{\partial \theta_{h}} \dot{\theta}_{h}+C_{Q h v} \frac{\partial \Psi_{Q h v}}{\partial a_{\theta h}} \frac{\partial a_{\theta h}}{\partial \theta_{j}} \dot{\theta}_{j}+\dot{C}_{S h \mu} \Psi_{S h \mu}+\dot{C}_{Q h \nu} \Psi_{Q h v}-R_{Q h}+ \\
& \left.+\sum_{m \in I_{O}} W_{Q h m}\right\}+\sum_{m \in I_{O}} \sum_{h^{\prime} \in I_{G}} I_{h^{\prime} m q k r} \ddot{Q}_{k r} \dot{Q}_{m q}+\sum_{m \in I_{o}} \sum_{h^{\prime} \in I_{G}}\left(\frac{\partial V_{S h}}{\partial Q_{m q}}-\bar{f}_{S h m q}\right) \dot{Q}_{m q}=0
\end{aligned}
$$

where additional terms dependent on $Q_{m q}$ are taken into account.

We would like to introduce friction terms as well as effects following from stochastic forces as a result of molecule - solvent interaction. Thus we assume existence, on the dimensionally reduced level, of additional forces $\bar{c}_{h q} w_{h q}$. Furthermore, the function $\Phi$ can be modified by averaging of effects of the stochastic forces. This is postulated by extension of the function $\Phi$ into the function $\Phi_{E}=\Phi+\Phi_{s}$ in (20). $\Phi_{s}$ depends on $Q_{h q}$ only for this derivation. This function will be plotted with variables $\theta, \eta, \eta_{S}$ in the sequel, where $\eta_{\mathrm{s}}$ is an internal variable which takes into account solvent properties during evolution of the system.

Let us assume that time processes are independent. Then we obtain the equations describing slowly varying processes

$$
\begin{gathered}
C_{S h \mu} \frac{\partial \Psi_{S h \mu}}{\partial Q_{h q}}+\sum_{h^{\prime}} C_{S h^{\prime} \mu} \frac{\partial \Psi_{S h^{\prime} \mu}^{r}}{\partial a_{Q h^{\prime}}} \frac{\partial a_{Q h^{\prime}}}{\partial Q_{h q}}+ \\
+\frac{\partial \Phi_{S}}{\partial Q_{h q}}+\bar{c}_{h q} w_{h q}-\bar{f}_{S h q}+M_{h p q} \dot{w}_{h p}+\sum_{h^{\prime}} I_{h^{\prime} h q k r} \ddot{Q}_{k r}=0
\end{gathered}
$$

with the additional condition

$$
\sum_{h} \frac{\partial V_{S h}}{\partial Q_{m q}}+\sum_{h} I_{h m q k r} \ddot{Q}_{k r}=\sum_{h} \bar{f}_{S h m q} \equiv \bar{f}_{S m q}, h \in I_{G}, \quad m \in I_{O} .
$$

We introduce also the equation which expresses previously introduced notation in the form

$$
\dot{Q}_{h q}=w_{h q}, h \in I_{G}
$$

The last term in (33) contains second time derivatives of $Q_{k r}$ with index $k$ which can be found in $I_{o}$. This induces necessity of considering furthermore the following additional condition

$$
\dot{Q}_{m q}=\bar{w}_{m q}, \quad m \in I_{O}
$$

for determining values of $\dot{Q}_{m q}$ which do not belong to the system $I_{G}$. 
We obtain also from (32), using (33) and (34), the equation describing averaged evolution of quickly varying processes

$$
\begin{aligned}
& \sum_{h}\left(C_{Q h \nu} \frac{\partial \Psi_{Q h v}}{\partial \theta_{h}} \dot{\theta}_{h}+C_{Q h \nu} \frac{\partial \Psi_{Q h v}}{\partial a_{\theta h}} \frac{\partial a_{Q h}}{\partial \theta_{j}} \dot{\theta}_{j}+\right. \\
& \left.+\dot{C}_{S h \mu} \Psi_{S h \mu}+\dot{C}_{Q h \nu} \Psi_{Q h v}-R_{Q h}+\sum_{m \in I_{O}} W_{Q h m}\right)=0
\end{aligned}
$$

with the additional condition

$$
W_{Q h m}=\bar{W}_{Q h m}, \quad h \in I_{G}, \quad m \in I_{O}
$$

motivated by considerations related to (6).

Since $I_{G}$ is not defined precisely, we assume that equations (37), (38) are valid for all $I_{G}$ and thereby for all parts of the molecule composed from distinguished subsystems. This necessitates in turn defining a family of conditions of type (38).

Let $\mathcal{H}_{h}=\left\{\mathbf{Q}_{h}, a_{Q h}, \theta_{h}, a_{\theta h}\right\}$. Above introduced equations have an excessive number of variables. Therefore, additional constitutive equations must be introduced. They are assumed in the following form

$$
\begin{gathered}
V_{S h}=C_{S h \mu}\left(\mathbf{C}_{S h \mu}, \mathcal{H}_{h}, \eta_{h}, \eta_{S}\right) \Psi_{S h \mu}\left(\mathbf{Q}_{h}, a_{Q h}\right), \\
V_{Q h}=C_{Q h v}\left(\mathbf{C}_{Q h v}, \mathscr{H}_{h}, \eta_{h}, \eta_{S}\right) \Phi_{Q h v}\left(\theta_{h}, a_{\theta h}\right), \\
\bar{W}_{Q h m}=\bar{W}_{Q h m}\left(\mathbf{C}_{W h}, \mathscr{H}_{h}, \eta_{h}, \eta_{S}\right), \\
\dot{\eta}_{h}=A_{\eta}\left(C_{\eta h}, \mathcal{H}_{h}, \eta_{h}, \eta_{S}\right),
\end{gathered}
$$

where $\eta_{s}$ represents additional internal state variables which describe effects of interactions of the molecule with solvent. We introduce also the evolution equation for these internal state variables by

$$
\dot{\eta}_{S}=A_{S}\left(\mathbf{C}_{S}, \mathscr{H}_{h}, \eta_{h}, \eta_{S}\right)
$$

Equations (33)-(43) determine skeletal dynamical system for the reactive nanostructure. Identification of introduced constants should be realized by means of comparison of solutions of molecular dynamics equations andnSDS equations. The comparison of solutions can be carried out by means of a metric $\rho_{D R}: \bar{V}_{T} \times \bar{V}_{T} \rightarrow \mathrm{R}_{+} \cup\{0\}$ determined in space of processes $\bar{V}_{T}$. Then the function $H$ defined by 


$$
H\left(\varphi_{0}, \mathbf{f}\right)=\inf _{\mathbf{C} \in \rho_{D}} \rho_{\mathcal{C}}\left(\pi_{T}\left(\varphi\left(\varphi_{0}, \mathbf{f}\right)(t)\right), \mathbf{d}\left(\mathbf{C}, \pi\left(\varphi_{0}\right), \pi_{f T}(\mathbf{f})(t)\right)\right)
$$

where $\mathbf{d}$ is a solution of $S D S$ equations, should be weakly dependent on quantities $\varphi_{0}$, f related to elementary dynamical system for obtaining a good approximation. $C$ stands for a set of admissible constants $\mathbf{C}$.

A numerical example of realization of the dimensional reduction procedure for a chain of atoms, carried out in similar way as this is discussed above, has been discussed in [21],

\subsection{Chemical reactions with reactive nanostructures}

We distinguish two kinds of chemical reactions. The first one is related to reactions with particles which are not defined as reactive nanostructures. We would say that they influence behaviour of the nanostructure. As a result we are interested mainly in effects which they cause. Therefore, description of such reactions is suggested by terms responsible for external interactions. Consequently, we introduce the following additional equations

$$
\begin{gathered}
\bar{f}_{S m q}=\bar{f}_{S m q}\left(\mathscr{H}_{h}, \eta_{h}, \eta_{S}, \eta_{R C}\right), m \in I_{O}, \\
\bar{W}_{Q h m}=\bar{W}_{Q h m}\left(\mathscr{H}_{h}, \eta_{h}, \eta_{S}, \eta_{R C}\right), m \in I_{O}, \\
\dot{\eta}_{R C}=A_{R C}\left(\dot{H}_{h}, \eta_{h}, \eta_{S}, \eta_{R C}\right),
\end{gathered}
$$

where $\eta_{R C}$ represents internal state variables which describe process of approaching, bonding and releasing of the external particles. They influence evolution of the system directly by $\bar{f}_{S m q}$ which can be viewed as a modifying factor related to $\Phi$ and also by interchange of energy expressed by $\bar{W}_{\text {Ohm }}$

The second kind of chemical reactions is related to joining of various reactive nanostructures as well as various forms of reorganization or fragmentation within a system of them. Then, we are interested in description of more detailed mechanisms governing such processes. Therefore, the notion of the molecular recognition should be transferred from atomic level of description into the nanoscale description.

We consider the reaction $a+b \rightarrow a b$ denoted by $R$ as in Definition 5 , and $\overline{C_{R}}$ is corresponding catchment region defined on the reduced level of description.

Let us consider a set $\bar{U}_{S} \subset \overline{\mathcal{V}}_{D}, \overline{\mathbf{D}}=\{\mathbf{Q}, \mathbf{w}\}$ and $\overline{\mathbf{D}}_{0} \in \bar{U}_{S}$ stand for an initial condition for evolution of $\tilde{\mathrm{D}}$. Let $\lambda(\mathrm{t})=\left\{\theta, \eta, \eta_{S}\right\}(t)$ stand for a process related to evolution of the remaining variables which have an effect on form of $\Phi$ and thereby also on the corresponding catchment region $\bar{C}_{R}$. Consequently, together with evolution of $\lambda$ we consider also evolution of $\bar{C}_{R}(\lambda)$. 
We define the set. $\mathcal{A}\left(\bar{U}_{S}\right)$ as a set of all process $\lambda(\mathrm{t})$ which do not change qualitatively the catchment region $\bar{C}_{R}(\lambda(\mathrm{t}))$ during evolution and $\overline{\mathbf{D}}\left(\overline{\mathbf{D}}_{0}, t\right) \in \bar{U}_{S}$ for each $t$ and $\lambda(\mathrm{t})$ considered. This condition ensures that processes $\lambda(\mathrm{t})$ do not disturb realization of the reaction $\mathscr{R}$.

We introduce below definition of molecular recognition which is associated with nanoscale level of modelling. This definition is not equivalent to the Definition 5. Difference rests on using now more averaged description. As a result we have lost some properties of molecules following from the averaging. Thereby, the molecular recognition defined here is not so precise as that one given in Definition 5.

\section{Definition 9:}

We say that two molecules $a$ and $b$ undergo the molecular recognition with respect to reaction $\Re$ with probability $p$, if they find themselves in a state $\overline{\mathbf{D}}_{0} \in \bar{U}_{S}$, where $\bar{U}_{S} \subset \overline{\mathcal{V}}_{D}$ is a maximal set satisfying the condition that there exists $t_{k}$ such thatfor each $\overline{\mathbf{D}}_{0} \in \bar{U}_{S}$ we have $\mathscr{P}\left(\pi_{C}\left(\overline{\mathrm{D}}\left(\overline{\mathrm{D}}_{0}\right.\right.\right.$ $\mathcal{L}(t))(t)) \in \bar{C}_{R}(\lambda(t)) \wedge\left\{\theta, \eta, \eta_{S}\right\}(t) \in \mathscr{A}\left(U_{S}\right)=p \leq 1$ and $\mathcal{P}_{i s}$ a probability measure related to this problem. The set $\vec{U}_{S}$ is called the range of selection.

The probability $p$ and stochastic forces. $\mathcal{L}(t)$ applied in this definition express connections with solvent interactions. It also means that we admit a stochastic effects in equations describing evolution of the nanostructure.

The range of selection enables to formulate conditions for realization of the reaction $R$ expressed in terms of the nanoscale model.

Form of the set $\bar{U}_{S}$ could be also considered as a phenomenological postulate. Then, phenomenological, nanoscale models of assembling or reorganization of reactive nanostructures could be formulated neglecting detailed atomic processes.

Let us notice that nanoscale models are perhaps very convenient in description of molecular processes using geometrical methods. Geometry of the potential energy hypersurface is intensively exploited for description of chemical reactions at the atomic level. The terms such as the steepest descent path and the catchment region are introduced just for the potential energy [14, 15]. However, dynamics of atomic nuclei does not admit considering the steepest descent path strictly as a trajectory of a chemical reaction. Furthermore, jumps through energetic barriers are admissible owing to this dynamics. Such dynamics is not represented by steepest descent paths which tends directly to a minimum.

Let us notice that this situation could be changed by some approximations related to the nanoscale model. First, discussed. $S Q$-decomposition averages thermal vibrations. Then, the energetic barriers which are jumped owing to thermal vibrations should vanish in description with the help of $\Phi_{\mathrm{E}}$. This means that the geometrical steepest descent path defined for $\Phi_{\mathrm{E}}$ could be viewed also as a path of a dynamical process. Furthermore, we could admit approximation that frictional constants are relatively big in comparison with inertia characteristics. For larger molecules in solution such approximation seems to be admissible. Then, the equation of dynamics of the reactive nanostructure (33) could be approximated by 


$$
\bar{c}_{h q} w_{h q}=-\frac{\partial \Phi_{E}}{\partial Q_{h q}}\left(\theta, \eta, \eta_{S}, \eta_{R C}\right)+\bar{f}_{S h q} .
$$

Thereby, geometry of $\Phi_{\mathrm{E}}$ could be more important in this description than the potential energy hypersurface geometry in atomic description. Evidently, some complications have to appear as a result of evolution of the remaining variables $\theta, \eta, \eta_{S}, \eta_{R C}$. In particular these variables affect the molecular recognition process.

Description of processes by means of nanostructures is characterized by a reduced number of degrees of freedom in comparison with molecular dynamics. However, direct connection between these approaches is maintained here by the dimensional reduction procedure. Thus, if we have equations for evolution of the molecular nanostructure derived by means of the molecular dynamics then we have also corresponding dimensional reduction procedure. This means that direct connection with molecular dynamics is expressed by mappings transforming variables, forces, form of the skeletal dynamical system and identification or approximation methods applied. As a result we obtain a complicated picture associated with derivation of discussed equations with the help of molecular dynamics. In such a case obtained equations can also be interpreted as a set of results of molecular dynamics calculations gathered in the dimensionally reduced equations.

Some processes are associated with multifunctional molecules. Let us mention for instance the transcription factor TFIIH which takes part in general transcription process and also in nucleotide excision repair [22]. Consequently, we can imagine that some molecules can cooperate within various complexes of other molecules taking part in corresponding various processes.

In order to describe such a situation probably changing dimensional reductions have to be applied in derivation of nanoscale models. This is so since we expect different external conditions acting on the molecule during evolution between mentioned stages. Consequently, we are interested in knowledge of range of validity of equations obtained by the dimensional reduction procedure. Furthermore, important properties of biological processes appear in the case of perturbation of them. Then, the perturbation could also cause exceeding admissible conditions for application of a given model.

Above discussion suggests that change of equations applied, during simulations of various stages of processes is probably an inherent property of modelling more complex processes. Consequently, some critical conditions for validity of currently applied model should be defined. Let us assume that a critical condition for a given model connected with the dimensional reduction procedure $D R^{\alpha}$ of $\alpha$ type takes the form

$$
K_{C R C}^{\alpha}\left(\mathbf{Q}_{h}, \dot{\mathbf{Q}}_{h}, \theta_{h}, \eta_{h}\right)=\bar{K}^{\alpha},
$$

where $\vec{K}^{\alpha}$ is a determined critical value.

If evolution of variables of the dynamical system associated with $D R^{\alpha}$ leads to violation of the condition (49) then we have to realize change of model corresponding to the dimensional reduction $D R^{\alpha}$ into that one which corresponds to $D R^{\alpha+1}$ Application of the new equations 
needs also determination of new initial conditions for variables associated with $D R^{\alpha+1}$. We assume these initial conditions in the form

$$
\mathbf{d}^{\alpha+1}\left(t_{0}^{\alpha+1}\right)=\Gamma^{\alpha, \alpha+1}\left(\mathbf{d}^{\alpha}\left(t_{f}^{\alpha}\right)\right)
$$

where $\mathbf{d}^{\alpha+1}, \mathbf{d}^{\alpha}$ are variables of dynamical systems associated with $D R^{a+1}$ and $D R^{a}$ correspondingly. $t_{f}^{\alpha}$ is a final instant of process described by means of $D R^{\alpha}$ and $t_{0}^{a+l}=t_{f}^{\alpha}$ determines an initial instant associated with $D R^{a+1} . I^{a, a^{a+1}}$ transforms the first type of variables into the second one.

Consequently, satisfactory result of modelling by dimensional reduction procedure consists of equations of the reduced dynamical system based on EDS and range of validity of these equations, expressed by some critical conditions. Then, we are able to realize change of equations during numerical simulations of multimolecular processes.

\subsection{Molecular motors}

Molecular motors are objects intensively investigated in biology during last years. These molecular systems transform chemical energy into mechanical one enabling realization of various kinds of motion. Let us mention kinesin as a molecule transporting cell organelles along microtubule [23], RNA polymerase which takes part in transcription [24] or $F_{1}$ molecular motor associated with synthesis of ATP [25]

Mathematical modelling of molecular motors is also the subject of many papers, especially in physical literature [26-28].

The question is whether we are able to discriminate a category of molecular motors within molecular reactive nanostructures. Thus, the aim of this section is to show that the molecular motors can be described within the suggested kind of a formal system.

It seems that nanoscale description is very convenient just for modelling molecular motors since thermal vibrations are averaged and then structural transformations of the molecule are of primary importance in this kind of modelling.

We consider two molecular systems. The first one is the molecular motor $S_{M T}$ and the second one is a supporting molecule $S_{S M}$ on which the molecular motor moves.

Configuration of the whole system is given by $\left\{\mathbf{Q}_{M T}, \mathbf{Q}_{S M}\right\} \in \mathcal{V}_{M T} \times V_{S M}=V_{M}$. Process of motion of the molecular motor is described by a path $\left\{\mathbf{Q}_{M T}, \mathbf{Q}_{M S}\right\}(\mathrm{s})$ and especially by the subpath $Q_{M T}^{\left({ }^{(s)}\right.}$. Motion is associated with varying function $\Phi_{E}=\Phi_{M}, \Phi_{M}: V_{M} \rightarrow R$ which describes also effects of interactions with solvent in accordance with discussion from previous section. Changes of $\Phi_{M}$ are induced by additional external reactions providing energy to the system $S_{M}=S_{M T} \cup S_{S M}$. Description of effects induced by these reactions is introduced by internal state variables $\eta_{R C}$ in accordance with (45-47).

Way of evolution of $\Phi_{M}$ can be characterized by forms of the catchment regions. These forms are dependent on internal state variables $\eta_{R C}$ responsible for evolution of external reactions. We introduce a parameter $s \in\left[\mathrm{s}_{l}, s_{F}\right]$ which characterizes a stage of the system in one cycle associated 
with energy supply connected with $\eta_{R C}$. The stage is determined by means of geometrical considerations related to evolving structure of the system.

Evolution of $\eta_{R C}$ leads to transformation of the catchment region by means of $\bar{f}_{S m q}=$ $\bar{f}_{S m q}\left(\eta_{R C}\right)$ which influences direct transformation of $\Phi_{M}$ as well as $\bar{W}_{Q h m} \subset \bar{W}_{Q h m}\left(77_{\mathrm{RC}}\right)$ which influences energetic state of the molecule and, as a result, also values of $\theta, \eta, \eta_{S}$.

We can discuss various forms of transformation of the catchment region. In case of elastic deformation of molecules induced by external interactions we assume that the catchment region is deformed without any qualitative changes. However, some processes are associated with two equilibrium positions and transformation between them. Let us discuss such a transformation.

We assume that between time instants $t_{\alpha}$ and $t_{T}$ the catchment region $C_{\alpha}$ with minimum $\boldsymbol{c}_{\alpha}$ is transformed into a new catchment region $\tilde{C}_{\beta}$ which contains, at this moment, a new minimum $\mathbf{c}_{\beta}$ and also the previous configuration $\boldsymbol{c}_{\alpha}$. Then, evolution of the configuration $\mathbf{c}_{\alpha}$ to $\mathbf{c}_{\beta}$ along the steepest descent path is realized. Such a situation can be interpreted as resulting from joining of some external, energy reach molecule to the system $S_{M}$. This effect is described by $\eta_{R C}$. Attaining of the point $\mathbf{c}_{\beta}$ by configuration of the molecule induces further evolution of $\eta_{R C}$ started at an instant $t_{R}$ and corresponding to transformation of the catchment region to the new form $\boldsymbol{c}_{\beta}$ with minimum the same or close to $\mathbf{c}_{\beta}$, however without $\mathbf{c}_{\alpha}$. Discussed above transformation of catchment regions associated with external reactions is denoted by $\mathcal{T}\left(\mathrm{C}_{\alpha}\right)=C_{\beta}$.

We assume that a sequence of transformations of the catchment regions is realized during evolution of $\eta_{R C}$. The variable can represent effects following from reactions of binding ATP and various stages of hydrolysis realized in presence of the molecule $S_{M}$, for instance. Then, energy is provided into the system $S_{M}$ and various structural transformations are realizable. We express this fact by the sequence of the catchment region transformations cal $\left\{\mathcal{T}_{i}\left(\eta_{R C}\right)\right\}$. In particular, we can distinguish subsystems $S_{i} \subset S_{M}$ responsible for some distinguished transformations associated with fragmentation of some parts of subsystems, their structural transformations, and rebinding other parts of subsystems what finally enables realization of motion of the molecular motor.

Let us discuss temporarily the case when the molecule $S_{M T}$ is free. Then, we have the decomposition of the configurational space $\mathcal{V}_{M T}=H \times_{f} F_{R}$. Let $\mu\left(A_{R}, T\right): H \rightarrow H^{\prime}$ be rigid transformation of $H, \mu\left(A_{R}, T\right)\left(\boldsymbol{Q}_{H}\right)=\mathbf{Q}_{H}, \quad \boldsymbol{Q}_{H},=A_{R}\left(\boldsymbol{Q}_{H}\right)+T\left(\boldsymbol{Q}_{H}\right)$. It means that each $\mathbf{Q}_{H} \in H$ is transformed along the fiber $F_{R}$ into new manifold $H^{\prime}$ by means of $\mu$. The catchment region $C_{M T}$ associated with this molecule is defined by properties of the potential energy considered on $H$ since the rigid transformation does not change values of this function. Consequently, the mappings $\mu\left(A_{R}, T\right)$ transform rigidly also the catchment region.

The decomposition of $\mathcal{V}_{M T}$ depends on the form of the function $\Phi$ corresponding to the free molecule. However, when the molecule interacts, the function $\Phi$ depends on external parameters. Then, $\Phi(\mathbf{Q}) \neq \Phi\left(\mathbf{Q}^{\prime}\right)$ for $\mathbf{Q}^{\prime}=\mu(\mathbf{Q}), \mathbf{Q} \in H$ in general. Then, also catchment regions can vary.

We would like to describe properties of transition of the molecular motor from one catchment region to the other one as a result of supplying energy by external particles. We expect that the state of the molecular motor should be the same after each cycle discussed. Let us express this property. 
Let $\left.\pi_{M T}\left(\lambda\left(t_{I}\right)\right)\right|_{s_{I}}$ be the mapping which transforms the catchment region $C_{1}$ corresponding to phase of reaction described by $s_{I}$ into the configuration subspace of the molecular motor $\mathcal{V}_{M T}=\mathcal{V}_{M}$. Then, $\left.\pi_{M T}\left(\lambda\left(t_{I}\right)\right)\right|_{S_{l}}\left(C_{1}\right)=C_{M T 1}$. Similar transformation changes the catchment region $C_{2}$ by $\left.\pi_{M T}\left(\lambda\left(t_{F}\right)\right)\right|_{S_{F}}\left(C_{2}\right)=C_{M T 2}$, where $t_{I}$ and $t_{F}$ are time instants related to $s_{I}$ and $s_{F}$ respectively. We have also assumed that $\eta_{R C}\left(s_{I}\right)=\eta_{R C}\left(s_{F}\right)$ and therefore this variable is neglected in discussed below definition.

\section{Definition 10:}

Let $S_{M T}$ undergo evolution induced by a chemical reaction represented by a variables $\eta_{R C}$. We say that $S_{M T}$ is the cyclic molecular motor if its configuration transformsfrom the catchment region $C_{1}$ into $C_{2}$ during evolution in each cycle. Properties of transformations of $C_{1}$ into $C_{2}$ are associated with the sequence of transformations cal $\left\{T_{i}\right\}$. Furthermore we assume that there exist $A_{R}$ and Tfor which the following condition is satisfied

$$
\mu\left(A_{R}, T\right)\left(\pi_{M T}\left(\left.\lambda\left(t_{l}\right)\right|_{S_{l}}\right)\left(C_{1}\right)\right)=\pi_{M T}\left(\left.\lambda\left(t_{F}\right)\right|_{s_{F}}\right)\left(C_{2}\right)
$$

and furthermore $\Phi(\mu(\mathbf{Q}))=\Phi(\mathbf{Q})$ for all $\mathbf{Q} \in C_{M T 1}$. Variables determining the catchment regions represent the same statefor $s_{I}$ and $s_{F}$. Structure of the supporting molecule $S_{S M}$ ensures that configurational variables $Q_{S M}$ for initial and final instant are able to attain appropriate values for fulfilling the property (51).

Summarizing, we state that instants $t_{I}$ and $t_{F}$ indicate initial and final state of the molecular motor within one cycle. Projections of structure and state of the whole system on the molecular motor subsystem $S_{M T}$ is characterized by rigidly transformed set $C_{M T 1}$ into $\mathrm{C}_{\mathrm{Mt} 2}$. This means that absence of chemical reactions for $t_{I}$ and $t_{F}$ follows the same state and structure of the molecular motor neglecting a rigid motion. This also corresponds to readiness for repeating next cycle and continuing motion along $S_{S M}$.

Realizability of the processes of motion of the molecular motor is also associated with the molecular recognition. Then, corresponding range of selection $\bar{U}_{S}$ and the set of admissible processes $\mathscr{A}\left(\bar{U}_{S}\right)$ should be defined in similar way as in Definition 9.

By the Definition 10 we have characterized a category of cyclic molecular motors by their physical state and form of evolution. Thereby, we see that description of this important molecular structure observed in molecular biology is realizable within our formal system.

\section{STATUS OF THE PRESENT STAGE OF FORMULATION OF THE FORMAL SYSTEM}

In this section we discuss status of the present stage of formulation of the formal system and possible ways which follow from it for further development of this approach. 
Discussion of formal system, in general, can be carried out with respect to various aims. We can accentuate for instance formal mathematical aspects. Then, we make assumptions, introduce definitions and create various theorems together with their proofs. We would say that this is purely mathematical point of view.

Another approach is connected with applications. Then, we would like to obtain a theory which satisfactorily describes behaviour of a discussed object. Then, we encounter again various situations. We can know entirely this object taking into account its structure and physical laws determining its behaviour. Then, the theory has, from the very beginning, clear physical foundations that leads to stable assumptions and enables realization of a clear way for mathematical formalization.

However, we can also consider objects such as biological cell with its processes which is not entirely recognized. Then, constructing of the formal system has to be realized by stages of development connected with advances in our understanding the object as well as with current increasing other possibilities such as, for instance, attainable power of numerical simulations. In such a case more important is well fitting of assumptions to described reality than, at this stage, discussion of far advanced mathematical consequences. This is so since we expect that status of assumptions is not entirely stable yet and they will undergo modifications. Such a situation corresponds just to the formal system constructed in this paper.

A naturally arising method of following in constructing the formal system for describing evolution of biological structures seems to be an attempt to define the biological structures precisely. However, such a definition has to be immersed in a set of mathematical notions in order to be suitable for needs of our formal system. As a result, we should have clear picture what mathematical methods we can use in modelling. Furthermore, such methods have to be related to all physical aspects of biological systems in order to create precise definition. Such a situation corresponds rather to final stage of formulation of a formal system. Thereby, we have to do with a closed circle of problems. On the one hand we would like to give precise definition of a biological structure within a complex mathematical context. On the other hand we try to propose just what mathematical structure is suitable to this end. As a result, we cannot start with discussion of our formal system on the natural way considered.

We should decide how to break this circle. Existence of such a feedback in doing steps towards mathematical formulation suggests that satisfactory formal system can be attained by realization of a sequence of steps. We have decided here to break the circle of problems by characterization of biological processes considering first properties important for modeling only. Thus, multiscale aspects and integrity of biological systems are discriminated as the most important to this end. Furthermore, we confine ourselves, at this stage, to considering a range of physical aspects which corresponds to descriptions placed between molecular dynamics and continuum mechanics.

Functions of particular biological structures within a larger system of them, seem to be the most important for precise defining of the biological structures. Consequently, having in mind, in further stages of investigations, necessity of defining or classification of such structures 
within the formal system, we try to localize the mathematical description around objects which, at first sight, are a determinant of biological processes. It is estimated that such objects are connected with property called here as "multimolecular processes".

Such an approach suggests that we expect gradual development of defining and classification of biological structures, together with constructing parts of our formal system. Taking this into account we consider the mathematical approach which could be extended into models of new phenomena. Such a possibility is embodied in the dimensional reduction procedure considered within the collection of dynamical systems.

We have introduced a set of definitions which create foundations of our mathematical description. Introduced definitions are formal and we should comment why, despite of their formal form, they have connections with modelling biological structures.

We consider our biological structures as a physical system. Therefore, each definition is in fact related to methods of description of a physical process. Distinguishing application to biology consists in gathering of a set of physical processes which are present in biology and adapting methods of modelling to them. Suitable specification of such processes is the determinant which indicates that we are aimed at modelling just biological processes.

Present stage of formulation of the formal system induces some investigation programs. Realization of them can be viewed as a preparation to the next stage of development of our formal system.

Mathematical definition of the molecular recognition suggests development of modelling of reacting molecules in water on a way on which the range of selection set can be determined. Let us note that finding of state of molecules in the range of selection is a necessary condition for precise realization of the reaction. Thus, if we do not know such a set we are not able modelling precisely realized reactions. As a result modelling of biological processes needs development of the part of chemical reaction dynamics which describes approaching molecules taking especially into account propagation of interactions through water. Such a dynamics is related to distances between molecules, larger than usually considered in chemical reaction dynamics distances, corresponding to transition states.

The second investigation program is related to development of methods of constructing the nanoscale models of molecules. Such models should be elaborated by means of molecular dynamics dynamical systems treated as elementary dynamical systems within the dimensional reduction procedure. Important aspect of this modelling is range of validity of derived equations which enable to change model in the case when the present one becomes not sufficient. As it was discussed previously, determination of the range of validity of equations is a necessary condition for using such models in complex, evolving conditions which happens in biological structures.

Let us notice that nanoscale models can be viewed as a set of results of molecular dynamics simulations which are synthesized in the dimensionally reduced form. Thereby, above discussed concepts determine tasks for molecular dynamics which rely on obtaining a system of nanoscale models, by means of dimensional reduction procedure, applicable in simulation of molecular phenomena in an averaged way. 
We can also consider nanoscale models based on phenomenological postulates. We observe in papers on molecular biology creation of concepts how investigated experimentally molecular system works. This is expressed usually by a scheme which describes sequentially stages of evolution of the discussed system. See for example protein elongation process realized in ribosome [29], Perhaps, using phenomenological nanoscale models we would be able to create an additional, supplementary region for speculation how such molecular structures act. An advantage from such an activity would be making more close experimental results and theoretical description.

Above discussed directions of investigations could be summarized by several steps which could be done in order to start with modelling biological processes.

1. Elaboration of methods of constructing nanoscale models for some small, prototype molecules. The methods would be based on dimensional reduction procedure applied to molecular dynamics $E D S$, describing behaviour of these molecules.

2. Development of modelling interactions between molecules in water for the nanoscale description.

3. Modelling chemical reactions using the nanoscale models.

4. Elaboration of methods of determination of range of selection sets in order to describe integrity of chains of chemical reactions.

5. Application of obtained in points 1-4 methods and knowledge to selected biological molecules.

6. Modelling a selected multimolecular process in cell.

Summarizing, points 1-6 describe a way of attaining the possibility of modelling biological processes by nanoscale models. We notice that in order to realize these points a considerable engagement is necessary. Therefore, we do not illustrate our formalism by an example of modelling of a particular biological process.

Discussed above methods differ from those usually applied in modelling biological processes. We observe that, predominantly, during modelling, we try to introduce a simplification in order to obtain solvable equations in the possible simple form. The question is what for we have discussed above so complicated methods and why we introduce simplified, dimensionally reduced models if we have to use solutions of the elementary dynamical system.

We use $E D S$ for identification of dimensionally reduced equations. After this procedure, equations of $R D S$ become independent on solutions of $E D S$ and can be applied in arbitrary situations within their range of validity as some simplified models. We accentuate importance of range of validity of equations for numerical simulation of complex objects. This is justified by fact that changing of models during simulation of processes undergoing varying external conditions which happen in complex systems is inevitable. Consequently, methods of modelling discussed in this paper reflects aspiration for attaining possibility of simulations of biological processes having considerable complexity.

In this paper we do not discuss methods of modelling related directly to quantum mechanics. Accordingly, many fine processes is not described by the discussed formal system. In particular, 
we cannot consider quantum tunnelling of proton, for example. This is so since the first aim of this paper has been devoted to discussion of integrated molecular dynamics and continuum mechanics description. However, an open way for incorporation of new mathematical models is left. It is expressed by a freedom in determination of elementary dynamical systems. In particular such systems can be associated with quantum mechanical description. If we introduce the elementary dynamical system which describes single elementary particles such as electrons and nuclei then we are able to discuss for instance charge transfer or motion of protons in molecular systems, viewed as important processes in biology. However, in such a case particular methods of realization of dimensional reduction procedures have to be elaborated.

\section{FINAL REMARKS}

Mathematical methods applied in modelling biological structures which are observed in literature have attained a stage of development which suggests discussion on possibility of modelling whole cell as a system of molecular processes. This fact has been the main premise for formulation introduced here formal system. It is my conviction that such a formulation in its more advanced form could be a candidate to modelling the whole cell. Such aim could be attained gradually and have to be coordinated with knowledge provided by molecular biology as well as with increasing possibilities of numerical simulations.

It seems to be reasonable striving to construction the most simple model of cell perhaps supported considerably by phenomenological assumptions. Such a model would be a starting point for gradual improvements taking onto account more fine processes.

Summarizing, we have proposed above the formal system aimed at modelling biological processes in cell which induces, at this stage of its development, some investigation programs. Furthermore, construction of this formal system takes into account possibilities of extension it by incorporation models of more fine phenomena appearing in biological structures. It is also expected a progress in defining or in classification of particular biological structures or processes within the formal system, following together with realization of the investigation programs proposed.

\section{References}

[1] G. F. Oster, G. M. Odell, The mechanochemistry ofcytogels, Physica 12D, 333-350 (1984).

[2] R. Elber, M. Karpulus, Multiple conformational states of proteins: A molecular dynamics analysis ofmyoglobin, Science 235, 318-321 (1987).

[3] M. E. Tuckerman, G. J. Martyna, Understanding modern molecular dynamics: techniques and applications, J. Phys. Chem. 104, 159-178 (2000).

[4] C. Truesdell, A first course in rational continuum mechanics, Baltimore, Maryland 1972.

[5] H. Haken, Synergetics, Springer Verlag, Berlin, Heidelberg, New York 1977.

[6] C. Truesdell, W. Noll, The non-linear field theories of mechanics, Handbuch der Physik III/3, S.Flúgge (ed.), Springer 1965.

[7] W. G. Hoover, Molecular dynamics, Lecture Notes in Physics 258, Springer Verlag 1986.

[8] G. Cicotti, W. G. Hoover, Molecular dynamics simulations of statistical mechanical systems, Proc. of the Int. School of Phys. Enrico Fermi 1986. 
[9] M. Parinello, A. Rahman, Polymorphic transitions in single crystals: A new molecular dynamics method, J. Appi. Phys. 52, 12, $7182-7190$ (1981).

[10] J-M. Lehn, Supramolecular chemistiy - scope and perspectives, molecules, supra-molecules, and molecular devices, Angew. Chem., Int. Ed. Engl. 27, 89-112 (1988).

[11] J. Kaczmarek, Chemical recognition dynamics as a theoretical approach to chemical synthesis. Bull, of the Polish Academy of Sciences, Techn. Sci. 48, 12-27 (2000).

[12] P. E. Smith, B. M. Pettitt, Modelling solvent in biomolecidar systems, J. Phys. Chem. 98, 9700-9711 (1994).

[13] W. Kołos, Quantum chemistry, PWN, Warszawa 1978.

[14] P. G. Mezey, Potential energy hypersurface, Eisner Science Publishers B.V., 1987.

[15] P. G. Mezey, Catchment region partitioning ofenergy hypersurface, Theor. Chim. Acta (Berl..) 58, 309-330 (1981).

[16] M. C. Beckerle, Spatial control ofactin filament assembly: Lessons from listeria, Cell 95, 741-748 (1998).

[17] A. Hall, Rho GTPases and the actin cytoskeleton. Science 279, 509-514 (1998).

[18] K. E. Prehoda, D. J. Lee, W. A. Lira, Structure of the Enabled/VASP homology 1, domain-peptide complex: A key component in the spatial control ofactin assembly, Cell 97, 471-480 (1999).

[19] W. R. Rudnicki, G. Bakalai'ski, B. Lesyng, A mezoscopic model of nucleic acids. Part 1. Lagrangean and quaternion molecular dynamics, J. Biomol. Str. and Dyn. 17/6, 1097-1108, (2000).

[20] C. von Westenholtz, Differentialforms in mathematical physics, North- Holland 1981.

[21] J. Kaczmarek, A method of integration ofmolecular dynamics and continuum mechanics for solids, TASK Quaterly 6/2, 253-271 (2002).

[22] P. K. Bhatia, Z. Wang, E. C. Fredberg, DNA repair and transcription, Curr. Opin. in Gen. and Dev., 6, 146-150(1996).

[23] N. Hirokawa, Kinesin and dynein superfamily proteins and the mechanism of organelle transport, Science 279, 519-526 (1998).

[24] j. Gelles, R. Landick, RNA polymerase as a molecular motor, Cell 93, 13-16 (1998).

[25] K. Kinosita, Jr., R. Yasuda, H. Noji, S. Ishiwata, M. Yoshida, $F_{1}$-ATPase: A rotary motor made of a single molecule, Cell, 93, 21-24, 1998.

[26] F. Jülicher, A. Ajdari, J. Prost, Modelling molecular motors, Rev. Mod. Phys. 69, 1269-1281 (1997).

[27] C. R. Doering, W. Horsthemke, J. Riordan, Nonequilibrium fluctuation-induced transport, Phys. Rev. Lett. 72, 2984-2987 (1994).

[28] R. D. Astumian, M. Bier, Fluctuation Driven Ratchets: Molecular motors, Phys. Rev. Lett., 72, 1766-1769(1994).

[29] K. Abel, F. Jurnak, A complex profile ofprotein elongation: translating chemical energy into molecular movement, Structure 4, 229-238 (1996). 